\title{
THE CONE OF CURVES OF FANO VARIETIES OF COINDEX FOUR
}

\author{
ELENA CHIERICI AND GIANLUCA OCCHETTA
}

\begin{abstract}
We classify the cones of curves of Fano varieties of dimension greater or equal than five and (pseudo)index $\operatorname{dim} X-3$, describing the number and type of their extremal rays.
\end{abstract}

\section{INTRODUCTION}

A smooth complex projective variety is called Fano if its anticanonical bundle $-K_{X}$ is ample. The index of $X, r_{X}$, is the largest natural number $m$ such that $-K_{X}=m H$ for some (ample) divisor $H$ on $X$, while the coindex of $X$ is defined as $\operatorname{dim} X+1-r_{X}$.

Since $X$ is smooth, $\operatorname{Pic}(X)$ is torsion free and therefore the divisor $L$ satisfying $-K_{X}=r_{X} L$ is uniquely determined and called the fundamental divisor of $X$.

It is known that $0<r_{X} \leq \operatorname{dim} X+1$ and, by a theorem of Kobayashi and Ochiai [11, $r_{X}=\operatorname{dim} X+1$ if and only if $(X, L) \simeq\left(\mathbb{P}^{\operatorname{dim} X}, \mathcal{O}_{\mathbb{P}}(1)\right)$, and $r(X)=\operatorname{dim} X$ if and only if $(X, L) \simeq\left(\mathbb{Q}^{\operatorname{dim} X}, \mathcal{O}_{\mathbb{Q}}(1)\right)$.

Fano varieties of coindex two, which are called del Pezzo varieties, have been classified in 9 using the Apollonius method, i.e. proving that the linear sistem $|L|$ contains a smooth divisor and constructing a ladder down to the well-known case of surfaces.

The same method works for Fano varieties of coindex three, called Mukai varieties; in [16] Mukai announced the classification assuming the existence of a smooth member in $|L|$, and this was proved by Mella in [14.

Since the classification of Fano fourfolds is very far from being known, it is not possible to use Apollonius method to study Fano varieties of coindex four; however, with different techniques which involve the study of families of rational curves, it is possible to describe their structure, i.e. their cone of curves.

Families of rational curves are related to another invariant of a Fano variety, the pseudoindex $i_{X}$, introduced by Wiśniewski in [21, which is defined as the minimum anticanonical degree of rational curves on $X$.

The pseudoindex is related to the dimension and to the Picard number $\rho_{X}$ of a Fano variety by a conjecture of Mukai [15, which states that

$$
\rho_{X}\left(i_{X}-1\right) \leq \operatorname{dim} X
$$

equality holding if and only if $X \simeq\left(\mathbb{P}^{i_{X}-1}\right)^{\rho_{X}}$.

This conjecture has been recently proved in 1 for Fano fivefolds and for Fano varieties of pseudoindex $i_{X} \geq \frac{\operatorname{dim} X+3}{3}$ which admit a covering unsplit family of rational curves (this is always 
the case if $i_{X}=\operatorname{dim} X-3$ and $\operatorname{dim} X \geq 6$ ), so it provides a good bound on the Picard number of Fano varieties of pseudoindex $i_{X} \geq \operatorname{dim} X-3$ and dimension greater than four.

By definition, the pseudoindex is an integral multiple of $r_{X}$, so varieties of coindex four have pseudoindex $i_{X} \geq \operatorname{dim} X-3$; by the main result in [3] it is easy to prove that, for varieties of dimension $\geq 5$, Picard number $\geq 2$ and coindex four, index and pseudoindex coincide. For this reason the classification of the cone of curves of Fano varieties of coindex four and dimension $\geq 5$ can be regarded as a special case of the following

Theorem 1.1. Let $X$ be a Fano variety of dimension $n \geq 5$, pseudoindex $i_{X}=n-3$ and Picard number $\rho_{X} \geq 2$. Then $N E(X)$ is generated by $\rho_{X}$ rays.

More precisely, we have the following list of possibilities, where $F$ stands for a fiber type contraction, $D_{i}$ for a divisorial contraction whose exceptional locus is mapped to a $i$-dimensional subvariety and $S$ for a small contraction.

All cases are effective.

\begin{tabular}{|c|c|c|c|c|c|c|}
\hline $\operatorname{dim} X$ & $\rho_{X}$ & $R_{1}$ & $R_{2}$ & $R_{3}$ & $R_{4}$ & $R_{5}$ \\
\hline \multirow[t]{15}{*}{5} & \multirow[t]{7}{*}{2} & $F$ & $F$ & & & \\
\hline & & $F$ & $D_{0}$ & & & \\
\hline & & $F$ & $D_{1}$ & & & \\
\hline & & $F$ & $D_{2}$ & & & \\
\hline & & $F$ & $S$ & & & \\
\hline & & $D_{2}$ & $D_{2}$ & & & \\
\hline & & $D_{2}$ & $S$ & & & \\
\hline & \multirow[t]{5}{*}{3} & $F$ & $F$ & $F$ & & \\
\hline & & $F$ & $F$ & $S$ & & \\
\hline & & $F$ & $F$ & $D_{1}$ & & \\
\hline & & $F$ & $F$ & $D_{2}$ & & \\
\hline & & $F$ & $D_{2}$ & $D_{2}$ & & \\
\hline & \multirow[t]{2}{*}{4} & $F$ & $F$ & $F$ & $F$ & \\
\hline & & $F$ & $F$ & $F$ & $D_{2}$ & \\
\hline & 5 & $F$ & $F$ & $F$ & $F$ & $F$ \\
\hline \multirow[t]{5}{*}{6} & \multirow[t]{4}{*}{2} & $F$ & $F$ & & & \\
\hline & & $F$ & $D_{1}$ & & & \\
\hline & & $F$ & $D_{2}$ & & & \\
\hline & & $F$ & $S$ & & & \\
\hline & 3 & $F$ & $F$ & $F$ & & \\
\hline 7 & 2 & $F$ & $F$ & & & \\
\hline & & $F$ & $D_{2}$ & & & \\
\hline 8 & 2 & $F$ & $F$ & & & \\
\hline
\end{tabular}


Note that, under the stronger assumption that $r_{X}=\operatorname{dim} X-3$, a local description of the Fano-Mori contractions of $X$ has been achieved by Andreatta and Wiśniewski in [4, hence we expect that in this case it will be possible to go further in the direction of an effective classification.

The paper is organized as follows: in section two we collect basic material concerning FanoMori contractions and families of rational curves as well as some definitions and results in [1] which we will use extensively throughout the proof, while in section three we construct examples showing that all cases in our list are effective.

We then start the proof of theorem 1.1 the case $\operatorname{dim} X=5$, which is the hardest, is divided into two parts: in section four we deal with Fano fivefolds which admit a quasi-unsplit locally unsplit covering family, which turn out to have always a fiber type contraction, and in section five we study the remaining cases, proving the following

Theorem 1.2. Let $X$ be a Fano fivefold of pseudoindex two which does not have a covering quasi-unsplit locally unsplit family of rational curves; then $\rho_{X}=2$ and $X$ is the blow-up of $\mathbb{P}^{5}$ along a two-dimensional smooth quadric (a section of $\mathcal{O}(2)$ in a linear $\mathbb{P}^{3} \subset \mathbb{P}^{5}$ ), or along a cubic scroll $\left(\mathbb{P}_{\mathbb{P}^{1}}(\mathcal{O}(1) \oplus \mathcal{O}(2))\right.$ embedded in an hyperplane of $\mathbb{P}^{5}$ by the tautological bundle), or along a Veronese surface.

Finally, the last section contains the proof of theorem 1.1 in the easier case of varieties of dimension greater than five.

\section{BACKGROUND MATERIAL}

2.1. Extremal contractions. Let $X$ be a smooth complex projective variety of dimension $n$ and let $K_{X}$ be its canonical divisor.

By Mori's Cone Theorem the closure of the cone of effective 1-cycles into the $\mathbb{R}$-vector space of 1-cyles modulo numerical equivalence, $\overline{\mathrm{NE}(X)} \subset N_{1}(X)$, is locally polyhedral in the part contained in the set $\left\{Z \in N_{1}(X) \mid K_{X} \cdot Z<0\right\}$; an extremal face $\sigma$ of $X$ is a face of this locally polyhedral part and an extremal ray is an extremal face of dimension one.

Note that, if $X$ is a Fano variety, then $\overline{\operatorname{NE}(X)}=\mathrm{NE}(X)$ is polyhedral and any face of $\operatorname{NE}(X)$ is an extremal face.

To every extremal face one can associate a morphism to a normal variety; namely we have the following Contraction Theorem due to Kawamata and Shokurov:

Theorem 2.1. Let $X$ and $\sigma$ be as above. Then there exists a projective morphism $\varphi: X \rightarrow W$ from $X$ onto a normal variety $W$ which is characterized by the following properties:

i) for every irreducible curve $C$ in $X, \varphi(C)$ is a point if and only if the numerical class of $C$ is in $\sigma$;

ii) $\varphi$ has connected fibers. 
Definition 2.1. The map $\varphi$ of the above theorem is usually called the Fano-Mori contraction (or the extremal contraction) associated to the face $\sigma$. A Cartier divisor $H$ such that $H=\varphi^{*} A$ for an ample divisor $A$ on $W$ is called a good supporting divisor of the map $\varphi$ (or of the face $\sigma$ ). An extremal ray $R$ is called numerically effective, or of fiber type, if $\operatorname{dim} W<\operatorname{dim} X$, otherwise the ray is non nef or birational; the terminology is due to the fact that if $R$ is non nef then there exists an irreducible divisor $D_{R}$ which is negative on curves in $R$.

We usually denote with $E=E(\varphi):=\left\{x \in X \mid \operatorname{dim}\left(\varphi^{-1} \varphi(x)\right)>0\right\}$ the exceptional locus of $\varphi$; if $\varphi$ is of fiber type then of course $E=X$.

If the codimension of the exceptional locus of a birational ray $R$ is equal to one, the ray and the associated contraction are called divisorial, otherwise they are called small.

2.2. Families of rational curves. For this subsection our main reference is [12], with which our notation is coherent.

Let $X$ be a normal projective variety and let $\operatorname{Hom}\left(\mathbb{P}^{1}, X\right)$ be the scheme parametrizing morphisms $f: \mathbb{P}^{1} \rightarrow X$; we consider the open subscheme $\operatorname{Hom}_{b i r}\left(\mathbb{P}^{1}, X\right) \subset \operatorname{Hom}\left(\mathbb{P}^{1}, X\right)$, corresponding to those morphisms which are birational onto their image, and its normalization $\operatorname{Hom}_{b i r}^{n}\left(\mathbb{P}^{1}, X\right)$; the group $\operatorname{Aut}\left(\mathbb{P}^{1}\right)$ acts on $\operatorname{Hom}_{b i r}^{n}\left(\mathbb{P}^{1}, X\right)$ and the quotient exists.

Definition 2.2. The space Ratcurves ${ }^{n}(X)$ is the quotient of $\operatorname{Hom}_{b i r}^{n}\left(\mathbb{P}^{1}, X\right)$ by $\operatorname{Aut}\left(\mathbb{P}^{1}\right)$, and the space $\operatorname{Univ}(X)$ is the quotient of the product action of $\operatorname{Aut}\left(\mathbb{P}^{1}\right)$ on $\operatorname{Hom}_{b i r}^{n}\left(\mathbb{P}^{1}, X\right) \times \mathbb{P}^{1}$.

Definition 2.3. We define a family of rational curves to be an irreducible component $V \subset$ Ratcurves $^{n}(X)$.

Given a rational curve $f: \mathbb{P}^{1} \rightarrow X$ we will call a family of deformations of $f$ any irreducible component $V \subset$ Ratcurves $^{n}(X)$ containing the equivalence class of $f$.

Given a family $V$ of rational curves, we have the following basic diagram:

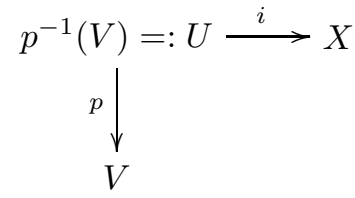

where $i$ is the map induced by the evaluation $e v: \operatorname{Hom}_{b i r}^{n}\left(\mathbb{P}^{1}, X\right) \times \mathbb{P}^{1} \rightarrow X$ and $p$ is a $\mathbb{P}^{1}$-bundle. We define $\operatorname{Locus}(V)$ to be the image of $U$ in $X$; we say that $V$ is a covering family if $\overline{\operatorname{Locus}(V)}=$ $X$. We will denote by $\operatorname{deg} V$ the anticanonical degree of the family $V$, i.e. the integer $-K_{X} \cdot C$ for any curve $C \in V$.

Given a family $V \subseteq \operatorname{Ratcurves}^{n}(X)$ and a point $x \in \operatorname{Locus}(V)$. we denote by $V_{x}$ the subscheme of $V$ parametrizing rational curves passing through $x$.

Definition 2.4. Let $V$ be a family of rational curves on $X$. Then

(a) $V$ is unsplit if it is proper; 
(b) $V$ is locally unsplit if for the general $x \in \operatorname{Locus}(V)$ every component $V_{x}$ of $V \cap$ Ratcurves $^{n}(X, x)$ is proper;

(c) $V$ is generically unsplit if there is at most a finite number of curves of $V$ passing through two general points of $\operatorname{Locus}(V)$.

Remark 2.1. Note that $(a) \Rightarrow(b) \Rightarrow(c)$.

Example 2.1. Let $R_{i}=\mathbb{R}_{+}\left[C_{i}\right]$ be an extremal ray such that the anticanonical degree of $\left[C_{i}\right]$ is minimal in $R_{i} ; C_{i}$ is often called a minimal extremal rational curve.

If we denote by $R^{i}$ an irreducible component of $\operatorname{Ratcurves}^{n}(X)$ containing $C_{i}$, then the family $R^{i}$ is unsplit: in fact, if $C_{i}$ degenerates into a reducible cycle, its components must belong to the ray $R_{i}$, since $R_{i}$ is extremal; but in $R_{i}$ the curve $C_{i}$ has the minimal intersection with the anticanonical bundle, hence this is impossible.

Proposition 2.1. (IV.2.6 in [12]) Let $X$ be a smooth projective variety and $V$ a family of rational curves.

Assume either that $V$ is generically unsplit and $x$ is a general point in $\operatorname{Locus}(V)$ or that $V$ is unsplit and $x$ is any point in $\operatorname{Locus}(V)$. Then

(a) $\operatorname{dim} X+\operatorname{deg} V \leq \operatorname{dim} \operatorname{Locus}(V)+\operatorname{dim} \operatorname{Locus}\left(V_{x}\right)+1$;

(b) $\operatorname{deg} V \leq \operatorname{dim} \operatorname{Locus}\left(V_{x}\right)+1$.

This last proposition, in case $V$ is the unsplit family of deformations of a minimal extremal rational curve, gives the fiber locus inequality:

Proposition 2.2. Let $\varphi$ be a Fano-Mori contraction of $X$ and let $E=E(\varphi)$ be its exceptional locus; let $S$ be an irreducible component of a (non trivial) fiber of $\varphi$. Then

$$
\operatorname{dim} E+\operatorname{dim} S \geq \operatorname{dim} X+l-1
$$

where

$$
l=\min \left\{-K_{X} \cdot C \mid C \text { is a rational curve in } S\right\} .
$$

If $\varphi$ is the contraction of a ray $R$, then $l$ is called the length of the ray.

Definition 2.5. We define a Chow family of rational curves to be an irreducible component $\mathcal{V} \subset \operatorname{Chow}(X)$ parametrizing rational and connected 1-cycles.

Given a Chow family of rational curves, we have a diagram as before, coming from the universal family over $\operatorname{Chow}(X)$.

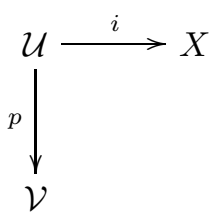


In the diagram $i$ is the map induced by the evaluation and the fibers of $p$ are connected and have rational components. Both $i$ and $p$ are proper (see for instance II.2.2 in [12]).

Definition 2.6. If $V$ is a family of rational curves, the closure of the image of $V$ in $\operatorname{Chow}(X)$ is called the Chow family associated to $V$.

Remark 2.2. If $V$ is proper, i.e. if the family is unsplit, then $V$ corresponds to the normalization of the associated Chow family $\mathcal{V}$.

Definition 2.7. Let $\mathcal{V}$ be the Chow family associated to a family of rational curves $V$. We say that $V$ is quasi-unsplit if every component of any reducible cycle in $\mathcal{V}$ is numerically proportional to $V$.

2.3. Notation. From now on we will denote with

$V$ a family of rational curves;

$\mathcal{V}$ the associated Chow family;

$[V]$ the numerical equivalence class in $N_{1}(X)$ of a general curve belonging to the family $V$;

$R_{i}$ an extremal ray of $X$;

$R^{i}$ the (unsplit) family of deformations of a minimal rational curve in $R_{i}$ (see example 2.1); $\varphi_{R_{i}}$ or $\varphi_{i}$ the extremal contraction associated with the ray $R_{i}$.

2.4. Chains of rational curves. For all the missing proofs in the rest of the section we refer the reader to 1 .

Let $X$ be a smooth variety, $\mathcal{V}^{1}, \ldots, \mathcal{V}^{k}$ Chow families of rational curves on $X$ and $Y$ a subset of $X$.

Definition 2.8. We denote by $\operatorname{Locus}\left(\mathcal{V}^{1}, \ldots, \mathcal{V}^{k}\right)_{Y}$ the set of points $x \in X$ such that there exist cycles $C_{1}, \ldots, C_{k}$ with the following properties:

- $C_{i}$ belongs to the family $\mathcal{V}^{i}$;

- $C_{i} \cap C_{i+1} \neq \emptyset$;

- $C_{1} \cap Y \neq \emptyset$ and $x \in C_{k}$,

i.e. $\operatorname{Locus}\left(\mathcal{V}^{1}, \ldots, \mathcal{V}^{k}\right)_{Y}$ is the set of points that can be joined to $Y$ by a connected chain of $k$ cycles belonging respectively to the families $\mathcal{V}^{1}, \ldots, \mathcal{V}^{k}$.

Note that $\operatorname{Locus}\left(\mathcal{V}^{1}, \ldots, \mathcal{V}^{k}\right)_{Y} \subset \operatorname{Locus}\left(\mathcal{V}^{k}\right)$.

Remark 2.3. If $Y$ is a closed subset, then $\operatorname{Locus}\left(\mathcal{V}^{1}, \ldots, \mathcal{V}^{k}\right)_{Y}$ is closed. 
Definition 2.9. Let $V^{1}, \ldots, V^{k}$ be unsplit families on $X$. We will say that $V^{1}, \ldots, V^{k}$ are numerically independent if their numerical classes $\left[V^{1}\right], \ldots,\left[V^{k}\right]$ are linearly independent in the vector space $N_{1}(X)$. If moreover $C \subset X$ is a curve we will say that $V^{1}, \ldots, V^{k}$ are numerically independent from $C$ if the class of $C$ in $N_{1}(X)$ is not contained in the vector subspace generated by $\left[V^{1}\right], \ldots,\left[V^{k}\right]$.

Lemma 2.1. Let $Y \subset X$ be a closed subset and $V$ an unsplit family. Assume that curves contained in $Y$ are numerically independent from curves in $V$, and that $Y \cap \operatorname{Locus}(V) \neq \emptyset$. Then for a general $y \in Y \cap \operatorname{Locus}(V)$

(a) $\operatorname{dim} \operatorname{Locus}(V)_{Y} \geq \operatorname{dim}(Y \cap \operatorname{Locus}(V))+\operatorname{dim} \operatorname{Locus}\left(V_{y}\right)$;

(b) $\operatorname{dim} \operatorname{Locus}(V)_{Y} \geq \operatorname{dim} Y+\operatorname{deg} V-1$.

Moreover, if $V^{1}, \ldots, V^{k}$ are numerically independent unsplit families such that curves contained in $Y$ are numerically independent from curves in $V^{1}, \ldots, V^{k}$ then either $\operatorname{Locus}\left(V^{1}, \ldots, V^{k}\right)_{Y}=\emptyset$ or

(c) $\operatorname{dim} \operatorname{Locus}\left(V^{1}, \ldots, V^{k}\right)_{Y} \geq \operatorname{dim} Y+\sum \operatorname{deg} V^{i}-k$.

Definition 2.10. We denote by $\operatorname{ChLocus}_{m}\left(\mathcal{V}^{1}, \ldots, \mathcal{V}^{k}\right)_{Y}$ the set of points $x \in X$ such that there exist cycles $C_{1}, \ldots, C_{m}$ with the following properties:

- $C_{i}$ belongs to a family $\mathcal{V}^{j}$

- $C_{i} \cap C_{i+1} \neq \emptyset$;

- $C_{1} \cap Y \neq \emptyset$ and $x \in C_{m}$,

i.e. $\operatorname{ChLocus}_{m}\left(\mathcal{V}^{1}, \ldots, \mathcal{V}^{k}\right)_{Y}$ is the set of points that can be joined to $Y$ by a connected chain of at most $m$ cycles belonging to the families $\mathcal{V}^{1}, \ldots, \mathcal{V}^{k}$.

Definition 2.11. We define a relation of rational connectedness with respect to $\mathcal{V}^{1}, \ldots, \mathcal{V}^{k}$ on $X$ in the following way: $x$ and $y$ are $\operatorname{in} \operatorname{rc}\left(\mathcal{V}^{1}, \ldots, \mathcal{V}^{k}\right)$-relation if there exists a chain of rational curves in $\mathcal{V}^{1}, \ldots, \mathcal{V}^{k}$ which joins $x$ and $y$, i.e. if $y \in \operatorname{ChLocus}_{m}\left(\mathcal{V}^{1}, \ldots, \mathcal{V}^{k}\right)_{x}$ for some $m$.

To the $\operatorname{rc}\left(\mathcal{V}^{1}, \ldots, \mathcal{V}^{k}\right)$-relation we can associate a fibration, at least on an open subset.

Theorem 2.2. ([5], IV.4.16 in [12]) There exist an open subvariety $X^{0} \subset X$ and a proper morphism with connected fibers $\pi: X^{0} \rightarrow Z^{0}$ such that

(a) the $r c\left(\mathcal{V}^{1}, \ldots, \mathcal{V}^{k}\right)$-relation restricts to an equivalence relation on $X^{0}$;

(b) the fibers of $\pi$ are equivalence classes for the $r c\left(\mathcal{V}^{1}, \ldots, \mathcal{V}^{k}\right)$-relation;

(c) for every $z \in Z^{0}$ any two points in $\pi^{-1}(z)$ can be connected by a chain of at most $2^{\operatorname{dim} X-\operatorname{dim} Z}-1$ cycles in $\mathcal{V}^{1}, \ldots, \mathcal{V}^{k}$.

Definition 2.12. In the above assumptions, if $\pi$ is the constant map we say that $X$ is $\mathrm{rc}\left(\mathcal{V}^{1}, \ldots, \mathcal{V}^{k}\right)$-connected. 
2.5. Bounding Picard numbers. In this subsection we list some conditions under which the numerical class (in $X$ ) of every curve lying in some subvariety $S \subset X$ is contained in a linear subspace of $N_{1}(X)$ or in a subcone of $\mathrm{NE}(X)$.

We write $N_{1}(S)=\left\langle\left[V^{1}\right], \ldots,\left[V^{k}\right]\right\rangle$ if the numerical class in $X$ of every curve $C \subset S$ can be written as $[C]=\sum_{i} a_{i}\left[C_{i}\right]$, with $a_{i} \in \mathbb{Q}$ and $C_{i} \in V^{i}$, and $\operatorname{NE}(S)=\left\langle\left[V^{1}\right], \ldots,\left[V^{k}\right]\right\rangle$ if the numerical class in $X$ of every curve $C \subset S$ can be written as $[C]=\sum_{i} a_{i}\left[C_{i}\right]$, with $a_{i} \in \mathbb{Q}_{\geq 0}$ and $C_{i} \in V^{i}$.

Lemma 2.2. (Lemma 1 in [18) Let $Y \subset X$ be a closed subset and $V$ an unsplit family of rational curves. Then every curve contained in $\operatorname{Locus}(V)_{Y}$ is numerically equivalent to a linear combination with rational coefficients

$$
\lambda C_{Y}+\mu C_{V}
$$

where $C_{Y}$ is a curve in $Y, C_{V}$ belongs to the family $V$ and $\lambda \geq 0$.

Corollary 2.1. Let $V$ be a family of rational curves and $x$ a point in $X$ such that $V_{x}$ is unsplit. Then $N_{1}\left(\operatorname{Locus}\left(V_{x}\right)\right)=N E\left(\operatorname{Locus}\left(V_{x}\right)\right)=\langle[V]\rangle$.

Corollary 2.2. Let $R_{1}$ be an extremal ray of $X, R^{1}$ a family of deformations of a minimal extremal curve in $R_{1}, x$ a point in $\operatorname{Locus}\left(R^{1}\right)$ and $V$ an unsplit family of rational curves, independent from $R^{1}$.

Then $N E\left(\operatorname{ChLocus}_{m}(V)_{\operatorname{Locus}\left(R_{x}^{1}\right)}\right)=\left\langle[V], R_{1}\right\rangle$.

Proof. Since

$$
\operatorname{ChLocus}_{m}(V)_{\operatorname{Locus}\left(R_{x}^{1}\right)}=\operatorname{Locus}(V)_{\operatorname{ChLocus}_{m-1}(V)_{\operatorname{Locus}\left(R_{x}^{1}\right)}},
$$

iterating lemma $2.2 m$ times any curve $C$ in $\operatorname{ChLocus}_{m}(V)_{\text {Locus }\left(R_{x}^{1}\right)}$ can be written as

$$
C \equiv \lambda C_{1}+\mu C_{V}
$$

with $\lambda \geq 0$, so we have only to prove that $\mu \geq 0$.

If $\mu<0$, then we can write $C_{1} \equiv \alpha C_{V}+\beta C$ with $\alpha, \beta \geq 0$; but since $C_{1}$ is extremal this implies that both $[C]$ and $\left[C_{V}\right]$ belong to $R_{1}$, a contradiction.

Remark 2.4. More generally, if $\sigma$ is an extremal face of $\operatorname{NE}(X), F$ is a fiber of the associated contraction and $V$ is an unsplit family independent from $\sigma$, the same proof shows that

$$
\operatorname{NE}\left(\operatorname{Locus}(V)_{F}\right)=\langle\sigma,[V]\rangle .
$$


2.6. Rational curves on Fano varieties. Let $X$ be a Fano variety and $\pi: X^{0} \rightarrow Z^{0}$ a proper surjective morphism on a smooth quasiprojective variety $Z^{0}$ of positive dimension.

By Theorem 2.1 in [13] we know that for a general point $z \in Z^{0}$ there exists a rational curve $C$ on $X$ of anticanonical degree $\leq \operatorname{dim} X+1$ which meets $\pi^{-1}(z)$ without being contained in it (an horizontal curve, for short).

We consider all the families containing these horizontal curves and, since they are only a finite number, we have that the locus of at least one of them dominates $Z^{0}$.

Definition 2.13. A minimal horizontal dominating family for $\pi$ is a family $V$ of horizontal curves such that $\operatorname{Locus}(V)$ dominates $Z^{0}$ and $\operatorname{deg} V$ is minimal among the families with this property. If $\pi$ is the identity map we say that $V$ is a minimal covering family.

Lemma 2.3. Let $X$ be a Fano variety, and let $\pi: X-\rightarrow Z$ be the rationally connected fibration associated to $k$ Chow families $\mathcal{V}^{1}, \ldots, \mathcal{V}^{k}$; let $V$ be a minimal horizontal dominating family for $\pi$. Then

(a) curves parametrized by $V$ are numerically independent from curves contracted by $\pi$;

(b) $V$ is locally unsplit;

(c) if $x$ is a general point in $\operatorname{Locus}(V)$ and $F$ is the fiber containing $x$, then

$$
\operatorname{dim}\left(F \cap \operatorname{Locus}\left(V_{x}\right)\right)=0 .
$$

Remark 2.5. Let $X$ be a Fano variety, $V^{1}, \ldots, V^{k}$ locally unsplit families of rational curves such that $V^{1}$ is covering and $V^{i}$ is horizontal and dominating with respect to the $\operatorname{rc}\left(\mathcal{V}^{1}, \ldots, \mathcal{V}^{i-1}\right)$ fibration; let $\pi: X--\rightarrow Z$ be the $\operatorname{rc}\left(\mathcal{V}^{1}, \ldots, \mathcal{V}^{k}\right)$-fibration. Then for general $x_{i} \in \operatorname{Locus}\left(V^{i}\right)$

$$
\sum \operatorname{dim} \operatorname{Locus}\left(V_{x_{i}}^{i}\right) \leq \operatorname{dim} X-\operatorname{dim} Z \text {. }
$$

Lemma 2.4. Let $X$ be a Fano variety, $V^{1}, \ldots, V^{k}$ locally unsplit families of rational curves such that $V^{1}$ is covering and $V^{i}$ is horizontal and dominating with respect to the $\operatorname{rc}\left(\mathcal{V}^{1}, \ldots, \mathcal{V}^{i-1}\right)$ fibration.

Let $\pi: X--\rightarrow Z$ be the $r c\left(\mathcal{V}^{1}, \ldots, \mathcal{V}^{k}\right)$-fibration and suppose that $\operatorname{dim} Z>0$.

Then either $\left[V^{1}\right], \ldots,\left[V^{k}\right]$ are contained in an extremal face of $N E(X)$ or there exists a small extremal ray $R$ whose exceptional locus is contained in the indeterminacy locus of $\pi$.

Proof. Since $X$ is normal and $Z$ is proper, the indeterminacy locus $E$ of $\pi$ in $X$ has codimension $\geq 2$ (see [1.39] in [6]). Take a very ample divisor $H$ on $Z$ and pull it back to $X$ : then $\pi^{*} H$ is zero on curves in $\left\langle\left[V^{1}\right], \ldots,\left[V^{k}\right]\right\rangle$ and it is non negative outside the indeterminacy locus of $\pi$. Therefore, either $\pi^{*} H$ is nef on $X$ and $\left\langle\left[V^{1}\right], \ldots,\left[V^{k}\right]\right\rangle$ lie on an extremal face of $\mathrm{NE}(X)$, or $\pi^{*} H$ is negative on an extremal ray, whose locus has to be contained in the indeterminacy locus of $\pi$ and therefore has codimension greater than one in $X$. 


\section{EXAmples}

In this section we show the effectiveness of all cases listed in theorem 1.1. If $X$ has only fiber type contractions, examples are given by the products such as $\mathbb{P}^{i_{X}-1} \times Y$, where $Y$ is a suitable fourfold of pseudoindex $i_{X}$ (for the ones with $i_{X}=2$ see [20]). The remaining cases are listed below:

\begin{tabular}{|c|c||c|c|c|c||c|}
\hline $\operatorname{dim} X$ & $\rho_{X}$ & $R_{1}$ & $R_{2}$ & $R_{3}$ & $R_{4}$ & \\
\hline \hline 5 & 2 & $F$ & $D_{0}$ & & & $\mathrm{a}$ \\
& & $F$ & $D_{1}$ & & & $\mathrm{~b}$ \\
& & $F$ & $D_{2}$ & & & $\mathrm{c}$ \\
& & $F$ & $S$ & & & $\mathrm{~d}$ \\
& & $D_{2}$ & $D_{2}$ & & & $\mathrm{e}$ \\
& & $D_{2}$ & $S$ & & & $\mathrm{f}$ \\
\hline & 3 & $F$ & $F$ & $S$ & & $\mathrm{~g}$ \\
& & $F$ & $F$ & $D_{1}$ & & $\mathrm{~h}$ \\
& & $F$ & $F$ & $D_{2}$ & & $\mathrm{i}$ \\
& & $F$ & $D_{2}$ & $D_{2}$ & & $\mathrm{j}$ \\
\hline & 4 & $F$ & $F$ & $F$ & $D_{2}$ & $\mathrm{k}$ \\
\hline \hline 6 & 2 & $F$ & $D_{1}$ & & & $\mathrm{l}$ \\
& & $F$ & $D_{2}$ & & & $\mathrm{~m}$ \\
& & $F$ & $S$ & & & $\mathrm{n}$ \\
\hline \hline 7 & 2 & $F$ & $D_{2}$ & & & $\mathrm{o}$ \\
\hline
\end{tabular}

a. $\quad X=\mathbb{P}_{\mathbb{P}^{4}}(\mathcal{O} \oplus \mathcal{O}(1))=B l_{p} \mathbb{P}^{5}$.

b1. $\quad X=\mathbb{P}_{\mathbb{P}^{3}}(\mathcal{O} \oplus \mathcal{O} \oplus \mathcal{O}(2))$.

b2. $\quad X=\mathbb{P}_{\mathbb{Q}^{3}}(\mathcal{O} \oplus \mathcal{O} \oplus \mathcal{O}(1))$.

c. $\quad X=\mathbb{P}_{\mathbb{P}^{2}}(\mathcal{O} \oplus \mathcal{O} \oplus \mathcal{O} \oplus \mathcal{O}(1))=B l_{\mathbb{P}^{2}} \mathbb{P}^{5}$.

d. $\quad X=\mathbb{P}_{\mathbb{P}^{3}}(\mathcal{O} \oplus \mathcal{O}(1) \oplus \mathcal{O}(1))$.

e1. Let $X=B l_{S_{3}} \mathbb{P}^{5}$, where $S_{3}$ is a cubic scroll contained in a hyperplane $H \subset \mathbb{P}^{5}$; denote by $\sigma$ the blow-up and by $E$ the exceptional divisor.

Let $\sigma^{*} \mathcal{O}_{\mathbb{P}^{5}}(1)$ be the pull-back to $X$ of the hyperplane bundle of $\mathbb{P}^{5}$, and let $\widetilde{H}=$ $\sigma^{*} \mathcal{O}(1)-E$ be the strict transform of $H$; the linear system

$$
|L|=\sigma^{*}\left|\mathcal{O}(2)-S_{3}\right|=\left|2 \sigma^{*} \mathcal{O}(1)-E\right|
$$

has empty base locus on $X$ and the associated map $\varphi_{|L|}$ gives $\widetilde{H}$ a structure of $\mathbb{P}^{2}$ bundle over $\mathbb{P}^{2}$.

Moreover $\widetilde{H}_{\mid \widetilde{H}}=\left(L-\sigma^{*} \mathcal{O}(1)\right)_{\mid \widetilde{H}}$, so that the restriction of $\widetilde{H}$ to each fiber of $\varphi_{|L|}$ is $\mathcal{O}_{\mathbb{P}^{2}}(-1)$; we can therefore apply the Nakano contractibility criterion 17, which yields the existence of a manifold $M \supset \mathbb{P}^{2}$ such that $X \simeq B l_{\mathbb{P}^{2}}(M)$ and $\widetilde{H}$ is the exceptional 
divisor of this blow-up.

Moreover, if we denote by $\psi$ the rational map associated to the linear system $\left|\mathcal{O}(2)-S_{3}\right|$ on $\mathbb{P}^{5}$ we have that the following diagram commutes:

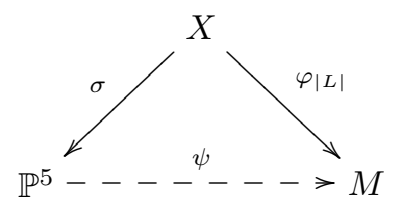

One can also prove (see [8] ) that $M$ is isomorphic to the hyperplane section associated with the Plücker embedding of the Grassmannian $G(1,4) \subset \mathbb{P}^{9}$, so (see [7.1] in [8]) $M$ is a del Pezzo variety.

Note that since $\rho_{X}=2$ and $X$ has two smooth blow-downs, $-K_{X}$ is positive on the entire cone $\operatorname{NE}(X)$, so $X$ is a Fano variety. Moreover, we can write

$$
-K_{X}=6 \sigma^{*} \mathcal{O}(1)-2 E=2\left(3 \sigma^{*} \mathcal{O}(1)-E\right),
$$

so $X$ has index 2 .

e2. Let $X=B l_{V} \mathbb{P}^{5}$, where $V$ is a Veronese surface. Denote by $\sigma$ the blow-up and by $E$ the exceptional divisor.

Consider on $\mathbb{P}^{5}$ the linear system $\left|\mathcal{O}_{\mathbb{P}^{5}}(2)-V\right|$ of the quadrics containing $V$, and denote by $\mathbb{P}^{5}-\stackrel{F}{-} \rightarrow \mathbb{P}^{5}$ the associated rational map; call $V^{\prime}$ the exceptional locus of $F^{-1}$ and let $\sigma^{\prime}: X^{\prime} \rightarrow \mathbb{P}^{5}$ be the blow-up of $\mathbb{P}^{5}$ along $V^{\prime}$.

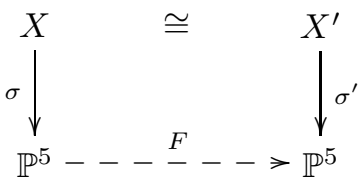

One can prove (see [2.0.2] in [7]) that $X^{\prime} \simeq X$, that the exceptional divisors of the two blow-ups satisfy the relations

$$
\begin{aligned}
& E=2 \sigma^{*} \mathcal{O}(1)-\sigma^{*} \mathcal{O}(1) \\
& E^{\prime}=2 \sigma^{\prime *} \mathcal{O}(1)-\sigma^{*} \mathcal{O}(1),
\end{aligned}
$$

and that the map $F$ is an involution (Theorem 2.6 in [7]).

As in the previous example, since $\rho_{X}=2$ and $X$ has two smooth blow-downs, $-K_{X}$ is positive on the entire cone $\operatorname{NE}(X)$, so $X$ is a Fano variety, and from the canonical bundle formula

$$
-K_{X}=6 \sigma^{*} \mathcal{O}_{\mathbb{P}^{5}}(1)-2 E=2\left(3 \sigma^{*} \mathcal{O}_{\mathbb{P}^{5}}(1)-E\right),
$$

we have that $X$ has index 2 . 
f. Let $X=B l_{\mathbb{Q}^{2}} \mathbb{P}^{5}$, where $\mathbb{Q}^{2} \subset \mathbb{P}^{5}$ is a smooth two-dimensional quadric, denote by $\sigma$ the blow-up and by $E$ the exceptional divisor; then

$$
-K_{X}=6 \sigma^{*} \mathcal{O}_{\mathbb{P}^{5}}(1)-2 E .
$$

For every curve $C \subset X$ which is not contained in $E$, we have that $\sigma(C)$ is a curve in $\mathbb{P}^{5}$ of a certain degree $d$, and the sum of the multiplicities of the points of intersection of $\sigma(C)$ and $\mathbb{Q}^{2}$ is $\leq 2 d$. This implies that

$$
-K_{X} \cdot C \geq 6 d-4 d \geq 2 d
$$

The exceptional divisor $E$ can be written as

$$
E=\mathbb{P}\left(\mathcal{N}_{\mathbb{Q}^{2} \mid \mathbb{P}^{5}}^{*}\right)=\mathbb{P}(\mathcal{O}(-2) \oplus \mathcal{O}(-1) \oplus \mathcal{O}(-1))
$$

and $E_{\mid E}=-\xi_{\mathcal{N}^{*}}$. If we denote by $Q$ the section of $\sigma: E \rightarrow \mathbb{Q}^{2}$ which corresponds to $\mathcal{O}(-2)$, then $\mathrm{NE}(E)=\left\langle\left[l_{1}\right],\left[l_{2}\right],\left[l_{3}\right]\right\rangle$, where $l_{1}$ and $l_{2}$ correspond to the two rulings of $Q$ and $l_{3}$ is a line in a fiber of $\sigma$.

If we write $K_{E}$ as $-3 \xi-6 \sigma^{*} \mathcal{O}_{\mathbb{Q}^{2}}(1)$, the adjunction formula yields

$$
-K_{X_{\mid E}}=-K_{E}+E_{\mid E}=2 \xi+6 \sigma^{*} \mathcal{O}(1)
$$

so $-K_{X} \cdot l_{i}=2$ for every $i$, hence $X$ is a Fano variety of pseudoindex 2 .

The line bundle $2 \sigma^{*} \mathcal{O}(1)-E$ is nef on $X$, and it vanishes on the strict transform of the $\mathbb{P}^{3} \subset \mathbb{P}^{5}$ which contains $\mathbb{Q}^{2}$; hence it is the supporting divisor of the small contraction of $\mathbb{P}^{3}$ to a point.

g. Let $\mathcal{E}=\mathcal{O}_{\mathbb{P}^{3}} \oplus \mathcal{O}_{\mathbb{P}^{3}}(1)$ and $Y=Z=\mathbb{P}_{\mathbb{P}^{3}}(\mathcal{E})=B l_{p} \mathbb{P}^{4}$; consider the fiber product $X=Y \times_{\mathbb{P}^{3}} Z$

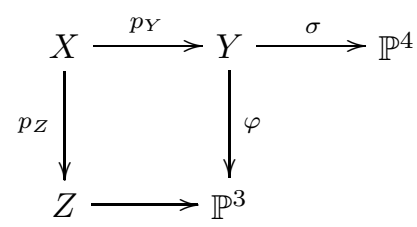

Call

$\xi_{Y} \in \operatorname{Pic}(Y)$ the tautological bundle of $\mathcal{E}$,

$H=\varphi^{*}\left(\mathcal{O}_{\mathbb{P}^{3}}(1)\right)$,

$E$ the exceptional divisor of $\sigma$,

$L=\sigma^{*}\left(\mathcal{O}_{\mathbb{P}^{4}}(1)\right)$.

Then

$$
\operatorname{Pic}(Y)=\left\langle\xi_{Y}, H\right\rangle=\langle E, L\rangle
$$

and the canonical bundle of $Y$ can be written as

$$
K_{Y}=-2 \xi_{Y}+\varphi^{*}\left(K_{\mathbb{P}^{3}}+\operatorname{det} \mathcal{E}\right)=-2 \xi_{Y}-3 H
$$


or

$$
K_{Y}=\sigma^{*} K_{\mathbb{P}^{4}}+3 E=-5 L+3 E .
$$

Note also that $\xi_{Y}=L$, and since for every fiber $f$ of $\varphi$ we have $H \cdot f=0, E \cdot f=L \cdot f=1$ and for every line $l \subset E$ which is not contracted by $\sigma$ we have $H \cdot l=1$, we can also write $H=L-E$.

Now consider on $Y$ the rank 2 vector bundle $\mathcal{F}=\mathcal{O}_{Y} \oplus H$ : then $X$ can be seen as $\mathbb{P}_{Y}(\mathcal{F})$. Call $\tilde{Y}$ the section of $p_{Y}$ which corresponds to the surjection $\mathcal{F} \rightarrow \mathcal{O}_{Y} \rightarrow 0$. If we denote by $\xi$ the tautological bundle of $\mathcal{F}$ we can write

$$
\begin{aligned}
-K_{X} & =2 \xi-p_{Y}^{*}\left(K_{Y}+\operatorname{det} \mathcal{F}\right) \\
& =2\left(\xi+p_{Y}^{*}\left(\xi_{Y}+H\right)\right),
\end{aligned}
$$

or, in terms of $L$ and $E$,

$$
-K_{X}=2\left(\xi+p_{Y}^{*}(2 L-E)\right) .
$$

First of all we show that $X$ is a Fano variety with $r_{X}=2$.

The line bundle $2 L-E$ is ample on $Y$, so $p_{Y}^{*}(2 L-E)$ is nef on $X$; since also $\xi$ is nef, we have to show that on every curve in $X$ at least one of these bundles is nonzero.

But the line bundle $p_{Y}^{*}(2 L-E)$ vanishes only on the fibers of $p_{Y}$, where we know that $\xi$ is positive, so $X$ is a Fano variety of index 2 .

$X$ has two fiber type contractions, associated with the nef bundles $p_{Y}^{*}(2 L-E)$ and $p_{Y}^{*} H+\xi$.

The line bundle $\xi+p_{Y}^{*} \xi_{Y}$ supports a small contraction which contracts a $\mathbb{P}^{3} \subset \widetilde{Y}$ to a point. In this case there exist two divisors on $X$ which are negative on the exceptional locus of this contraction: one is $\widetilde{Y}$ and the other is $p_{Y}^{*} E$ : in fact, since $E=L-H$, we have $p_{Y}^{*} E \cdot l=-1$ for every line $l \subset \mathbb{P}^{3}$.

h1. $\quad X=\mathbb{P}_{\mathbb{P}^{3}}(\mathcal{O} \oplus \mathcal{O}(2)) \times \mathbb{P}^{1}$.

h2. $\quad X=\mathbb{P}_{\mathbb{P}^{3}}(\mathcal{O} \oplus \mathcal{O}(1)) \times \mathbb{P}^{1}$.

h3. $\quad X=\mathbb{P}_{\mathbb{Q}^{3}}(\mathcal{O} \oplus \mathcal{O}(1)) \times \mathbb{P}^{1}$.

i. $\quad X=B l_{l} \mathbb{P}^{4} \times \mathbb{P}^{1}$.

j1. Here we construct an example of a fivefold where the two divisorial contractions have the same exceptional locus.

Let $\mathcal{F}=\mathcal{O}_{\mathbb{P}^{2} \times \mathbb{P}^{2}} \oplus \mathcal{O}_{\mathbb{P}^{2} \times \mathbb{P}^{2}}(1,1)$ and $X=\mathbb{P}_{\mathbb{P}^{2} \times \mathbb{P}^{2}}(\mathcal{F})$, let $\pi$ be the projection map, $\xi$ the tautological bundle on $X$ and $E$ the section of $\pi$ which corresponds to the surjection $\mathcal{F} \rightarrow \mathcal{O}_{\mathbb{P}^{2} \times \mathbb{P}^{2}} \rightarrow 0$. Then

$$
-K_{X}=2 \xi-\pi^{*}\left(K_{\mathbb{P}^{2} \times \mathbb{P}^{2}}+\operatorname{det} \mathcal{F}\right)=2\left(\xi+\pi^{*} \mathcal{O}(1,1)\right)
$$

since $\pi^{*} \mathcal{O}(1,1)$ vanishes only on the fibers $f$ of $\pi$, while $\xi \cdot f=1$, it follows that $X$ is a Fano variety and $i_{X}=2$. 
Obviously $X$ admits a fiber type contraction, which is given by its structure of $\mathbb{P}^{1}$ bundle and is supported by $\pi^{*} \mathcal{O}(1,1)$.

The nef bundles $\xi+\pi^{*} \mathcal{O}(1,0)$ and $\xi+\pi^{*} \mathcal{O}(0,1)$ vanish each on one "ruling" of $E$, so they support two different contractions of $E$ to $\mathbb{P}^{2}$, which are in fact smooth blowdowns.

Finally, the line bundles $\pi^{*} \mathcal{O}(1,0)$ and $\pi^{*} \mathcal{O}(0,1)$ support the contractions of the two faces of $\operatorname{NE}(X)$ which contain the fiber type ray, as shown in the diagram.

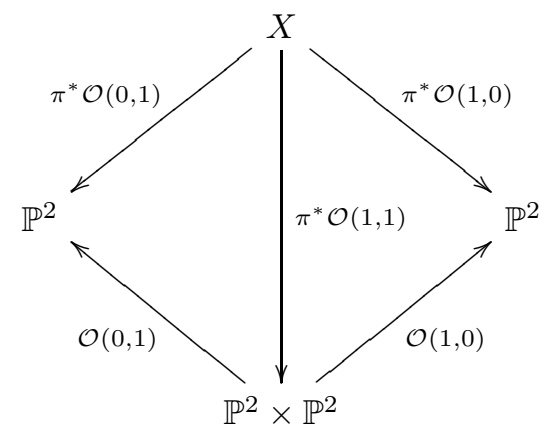

j2. An example of a fivefold with two divisorial contractions with disjoint exceptional loci is $X=B l_{\Pi_{1} \sqcup \Pi_{2}} \mathbb{P}^{5}$, the blow-up of $\mathbb{P}^{5}$ along two disjoint planes $\Pi_{1}, \Pi_{2}$.

Let $\sigma: X \rightarrow \mathbb{P}^{5}$ be the blow-up and denote by $E_{1}$ and $E_{2}$ the exceptional divisors; by the canonical bundle formula we have

$$
-K_{X}=6 \sigma^{*} \mathcal{O}_{\mathbb{P}^{5}}(1)-2 E_{1}-2 E_{2}=2\left(3 \sigma^{*} \mathcal{O}_{\mathbb{P}^{5}}(1)-E_{1}-E_{2}\right) .
$$

We want to prove that $H:=3 \sigma^{*} \mathcal{O}_{\mathbb{P}^{5}}(1)-E_{1}-E_{2}$ is ample on $X$ : if $C$ is a curve not contained in $E_{1} \cup E_{2}$, then $\sigma(C)$ is a curve of degree $d$ in $\mathbb{P}^{5}$ which intersects $\Pi_{1}$ and $\Pi_{2}$ in a number of points which has to be less or equal than $d$ (counted with multiplicity). So

$$
H \cdot C \geq d \geq 1 .
$$

As for curves contained in an exceptional divisor $E_{i}$, we know that

$$
E_{i}=\mathbb{P}_{\mathbb{P}^{2}}\left(\mathcal{N}_{\Pi_{i} \mid \mathbb{P}^{5}}^{*}\right)=\mathbb{P}\left(\mathcal{O}(-1)^{\oplus 3}\right) \simeq \mathbb{P}^{2} \times \mathbb{P}^{2}
$$

so

$$
-K_{E_{i}}=3 \xi_{i}+6 \pi^{*} \mathcal{O}_{\mathbb{P}^{2}}(1)
$$

where $\xi_{i}$ is the tautological bundle of $\mathcal{N}_{\Pi_{i} \mid \mathbb{P}^{5}}^{*}$ and $\pi$ is the projection $E_{i} \rightarrow \mathbb{P}^{2}$. By the adjunction formula, recalling that $-E_{i \mid E_{i}}=\xi_{i}$ we have that

$$
\left(-K_{X}\right)_{\mid E_{i}}=-K_{E_{i}}+E_{i \mid E_{i}}=3 \xi_{i}+6 \pi^{*} \mathcal{O}(1)-\xi_{i}=2 \xi_{i}+6 \pi^{*} \mathcal{O}(1),
$$

so $H_{\mid E_{i}}=\xi_{i}+3 \pi^{*} \mathcal{O}(1) \simeq \mathcal{O}_{\mathbb{P}^{2} \times \mathbb{P}^{2}}(1,-1) \otimes \mathcal{O}_{\mathbb{P}^{2} \times \mathbb{P}^{2}}(0,1)^{\otimes 3} \simeq \mathcal{O}_{\mathbb{P}^{2} \times \mathbb{P}^{2}}(1,2)$ which is ample, hence we have proved that $X$ is a Fano variety of index 2 . 
Now consider in $\mathbb{P}^{5}$ the lines which intersect both $\Pi_{1}$ and $\Pi_{2}$, and call $V$ the family of deformations of their strict transforms on $X$. Then $V$ is covering (since lines meeting $\Pi_{1}$ and $\Pi_{2}$ cover the whole $\mathbb{P}^{5}$ ) and $-K_{X} \cdot V=2$, so $V$ is unsplit. Hence $V$ is extremal and is associated to a fiber type contraction $\varphi: X \rightarrow Y$, which can be easily proved to be a $\mathbb{P}^{1}$-bundle over a smooth fourfold.

The fibers of $\varphi$ are the strict transforms of the lines meeting $\Pi_{1}$ and $\Pi_{2}$, hence, for any fiber $f$ we have $E_{i} \cdot f=1$; being $E_{1} \cap E_{2}=\emptyset$ it follows that $E_{1}$ and $E_{2}$ are sections of $\varphi$.

It's now easy to prove that $Y \simeq \mathbb{P}^{2} \times \mathbb{P}^{2}$ and $X=\mathbb{P}_{Y}(\mathcal{O}(1,2) \oplus \mathcal{O}(2,1))$.

j3. In this example the exceptional loci of the two divisorial contractions of $X$ are different but have nonempty intersection.

Let $Y=B l_{l} \mathbb{P}^{4}=\mathbb{P}_{\mathbb{P}^{2}}(\mathcal{O} \oplus \mathcal{O} \oplus \mathcal{O}(1))$.

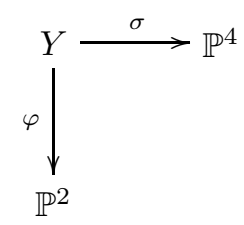

Call $H=\varphi^{*} \mathcal{O}_{\mathbb{P}^{2}}(1)$ and let

$$
X=\mathbb{P}_{Y}\left(H \oplus \mathcal{O}_{Y}(1)\right)
$$

Using the same notation as in example $\mathbf{g}$. we can write

$$
-K_{X}=2\left(\xi+\pi^{*}\left(\xi_{Y}+H\right)\right),
$$

so again we have that $X$ is a Fano variety of (pseudo)index 2 .

$X$ admits a fiber type contraction on $Y$, which is supported by $\pi^{*}(L+H)$, and two divisorial contractions: the first one contracts the special section on $X$ to $\mathbb{P}^{2}$ and is supported by $\xi+\pi^{*} H$, while the second one contracts the $\mathbb{P}^{1}$-bundle over the exceptional divisor in $Y$ to a two-dimensional quadric, and is supported by $\xi+\pi^{*} L$.

k. $\quad X=\mathbb{P}^{1} \times \mathbb{P}^{1} \times B l_{p} \mathbb{P}^{3}$.

l. $X=\mathbb{P}_{\mathbb{P}^{4}}(\mathcal{O} \oplus \mathcal{O} \oplus \mathcal{O}(1))=B l_{l} \mathbb{P}^{6}$.

m. $\quad X=\mathbb{P}_{\mathbb{P}^{3}}(\mathcal{O} \oplus \mathcal{O} \oplus \mathcal{O} \oplus \mathcal{O}(1))=B l_{\mathbb{P}^{2}} \mathbb{P}^{6}$

n. $\quad X=\mathbb{P}_{\mathbb{P}^{4}}(\mathcal{O} \oplus \mathcal{O}(1) \oplus \mathcal{O}(1))$.

o. $\quad X=\mathbb{P}_{\mathbb{P}^{4}}(\mathcal{O} \oplus \mathcal{O} \oplus \mathcal{O} \oplus \mathcal{O}(1))=B l_{\mathbb{P}^{2}} \mathbb{P}^{7}$ 


\section{FAno fivefolds With a COVERING QUASI-UNSPlit FAMILY}

In this section we start the proof of theorem 1.1. We know as a general fact (see subsection 2.6) that on $X$ there exist covering locally unsplit families of rational curves of degree $\leq$ $\operatorname{dim} X+1$; since we are assuming $\rho_{X} \geq 2$ these families have degree $\leq 5$, otherwise proposition 2.1 and corollary 2.1 would imply $\rho_{X}=1$.

We start considering the case when one of these families is quasi-unsplit. Note that this is the case if on $X$ there exists a fiber type ray $R$ : in fact, in this case, through every $x \in X$ there exists a rational curve which is contracted by $\varphi_{R}$ and has degree $\leq \operatorname{dim} X+1$; among the families of deformations of these curves we can choose a covering one with minimal degree, which is quasi-unsplit since $R$ is extremal.

Lemma 4.1. Let $V$ be a covering quasi-unsplit locally unsplit family of rational curves and $R_{1}$ an extremal ray of $N E(X)$ independent from $[V]$; assume that the contraction $\varphi_{R_{1}}$ has a three-dimensional fiber $F$. Then there exists a covering unsplit family which is numerically proportional to $V$.

Proof. If $V$ is not unsplit then $\operatorname{deg} V \geq 4$, so $V_{x}$ cannot be unsplit for any $x \in F$, otherwise we would have $\operatorname{dim}\left(\operatorname{Locus}\left(V_{x}\right) \cap F\right) \geq 1$ against the fact that $\mathrm{NE}\left(\operatorname{Locus}\left(V_{x}\right)\right)=\langle[V]\rangle$ and $\mathrm{NE}(F)=\left\langle R_{1}\right\rangle$.

Therefore through every point of $F \cap \operatorname{Locus}(V)$ there passes a reducible cycle in $\mathcal{V}$. Moreover, $\operatorname{Locus}(\mathcal{V})$ is closed since $\mathcal{V}$ is proper, and since $V$ is covering $\operatorname{Locus}(\mathcal{V})=X$, hence through any point of $F$ there is a reducible cycle in $\mathcal{V}$.

It follows that $F$ is contained in the locus of the family of deformations of one of the components of these cycles. Note that, since $\operatorname{deg} V \leq 5$ and $V$ is quasi-unsplit, such a family is unsplit and numerically proportional to $V$.

We denote this family by $\alpha V$, and applying lemma 2.1(a) we obtain that $\operatorname{dim} \operatorname{Locus}(\alpha V)_{F} \geq 5$, so $\alpha V$ is covering.

Lemma 4.2. Let $V$ be a covering unsplit family of rational curves and $R_{1}$ an extremal ray of $N E(X)$ independent from $[V]$. Assume that the contraction $\varphi_{R_{1}}$ has a three-dimensional fiber $F$ and let $D$ be an irreducible component of Locus $(V)_{F}($ note that, by lemma $2.1, \operatorname{dim} D \geq 4)$. Then

(a) if either $D=X$ or $D \cdot V>0$ then $N E(X)=\left\langle[V], R_{1}\right\rangle$;

(b) if $R_{2}$ is a birational ray different from $R_{1}$ then $D \cdot R^{2}=0$;

(c) if $R_{2}$ is a divisorial ray and $E_{2}$ is its exceptional locus, then $E_{2} \cdot V=E_{2} \cdot R^{1}=0 ;$

(d) if $R_{2}$ is a fiber type ray then $D \cdot R^{2}>0$.

Proof. The proof of (a) is an easy consequence of corollary 2.2, in fact, we know that $\mathrm{NE}(D)=$ $\left\langle[V], R_{1}\right\rangle$, and if $D$ is a divisor and $D \cdot V>0$ we can write $X=\operatorname{ChLocus}_{2}(V)_{F}$ (since $V$ is covering), while if $D=X$ the proof is trivial. 
To prove (b), we observe that the nontrivial fibers of $\varphi_{R_{2}}$ have dimension $\geq 2$, so if $D \cdot R^{2} \neq 0$ then $D$ contains a curve whose numerical class is in $R_{2}$, a contradiction.

In case (c), if either $E_{2} \cdot V>0$ or $E_{2} \cdot R^{1} \neq 0$ then $E_{2} \cap D \neq \emptyset$. Take a point $x \in E_{2} \cap D$ and a curve $C$ in $R^{2}$ passing through $x$ : by (c) we know that $D \cdot C=0$, so $C \subseteq D$, a contradiction. Finally, to prove (d) let $x$ be a point in $D$ and $C$ a curve in $R^{2}$ through $x$. Since $C$ cannot be contained in $D$ we must have $D \cdot R^{2}>0$.

Remark 4.1. Note that (b), (c) and (d) still hold if we replace $R^{2}$ with any noncovering unsplit family which is independent from $V$ and $R^{1}$.

Lemma 4.3. Let $R$ be a divisorial ray on $X$, let $E$ be its exceptional locus and consider the intersection number $E \cdot R^{i}$ with all the divisorial extremal rays of $N E(X)$ different from $R$. Then $E \cdot R^{i}<0$ for at most one index $i$; moreover in this case $E \cdot R^{j}=0$ for every $j \neq i$ and $N E(E)=\left\langle R, R_{i}\right\rangle$.

Proof. Assume that there exists an index $i$ such that $E \cdot R^{i}<0$; then we have $E=\operatorname{Locus}(R)_{\operatorname{Locus}\left(R_{x}^{i}\right)}$, so $\mathrm{NE}(E)=\left\langle R, R_{i}\right\rangle$ by corollary 2.2 In particular, $E$ cannot contain curves whose class is in $R_{j}$ for $j \neq i$.

We can now start the proof of theorem 1.1

$\boldsymbol{\rho}_{\boldsymbol{X}}=\mathbf{2}$. We have to prove that at least one of the extremal contractions on $X$ is of fiber type. Assume that this is not the case; in particular $[V]$ is not extremal, so by lemma 2.4 there exists a small extremal ray $R_{1}$.

Denote by $R_{2}$ the other extremal ray of $\operatorname{NE}(X)$; by lemma 4.1 we can assume that $V$ is unsplit, and by lemma 4.2 either $\mathrm{NE}(X)=\left\langle[V], R_{2}\right\rangle$ and $[V]$ is extremal or there exists an effective divisor $D$ such that $D \cdot V=D \cdot R^{2}=0$, implying that $D$ is numerically trivial on $\mathrm{NE}(X)$; in both cases we reach a contradiction.

$\rho_{X}=3$. We divide this part of the proof into three cases.

Case 1. All rays of $\mathrm{NE}(X)$ are of fiber type.

If two rays, say $R_{1}$ and $R_{2}$, do not lie on the same extremal face of $\operatorname{NE}(X)$, we can consider the rationally connected fibration $\pi: X-->Z$ associated to $R^{1}$ and $R^{2}$. Since $\rho_{X}=3$ we have $\operatorname{dim} Z>0$, so by lemma $2.4 X$ must have a small elementary contraction, a contradiction. The only possibility to exclude this situation is that $\mathrm{NE}(X)$ has exactly three rays.

Case 2. In $\mathrm{NE}(X)$ there exists a small extremal ray.

In this case we prove that $\mathrm{NE}(X)=\left\langle R_{1}, R_{2}, R_{3}\right\rangle$, where $R_{1}$ is small and both $R_{2}$ and $R_{3}$ are of fiber type.

Denote the small ray by $R_{1}$, and denote by $F_{1}$ an irreducible component of a fiber of $\varphi_{R_{1}}$. Note that by lemma 4.1 we can assume that $V$ is unsplit. 
First of all we prove that $X$ has at least one fiber type contraction: suppose that this is not the case, let $D_{1}=\operatorname{Locus}(V)_{F_{1}}$ and apply lemma 4.2. Since $\rho_{X}=3$ we cannot be in case (a), and so $D_{1}$ is a divisor such that $D_{1} \cdot R^{i}=0$ for every $i \neq 1$; as a consequence $\operatorname{NE}(X)=\left\langle R_{1}, R_{2}, R_{3}\right\rangle$. If $R_{2}$ is a small ray, we can repeat the same argument with the divisor $D_{2}=\operatorname{Locus}(V)_{F_{2}}$, and we obtain that $D_{2}$ vanishes on the face $\left\langle R_{1}, R_{3}\right\rangle$; since $D_{1}$ vanishes on the face $\left\langle R_{2}, R_{3}\right\rangle$ and $D_{1} \cdot V=D_{2} \cdot V=0$, it must be $\left[R^{3}\right]=[V]$, against the assumption that $X$ has no fiber type contractions.

So both $R_{2}$ and $R_{3}$ are divisorial. By lemma 4.2 (c), if we denote by $E_{i}$ the exceptional locus of $R_{i}$ we have $E_{i} \cdot V=E_{i} \cdot R^{1}=0$, and we know that $E_{i} \cdot R^{i}<0$, which implies $E_{2} \cdot R^{3}>0$ and $E_{3} \cdot R^{2}>0$; in particular this yields that the intersection numbers of $E_{2}$ and $E_{3}$ with every curve in $X$ have opposite signs. The existence of curves which intersect $E_{2} \cup E_{3}$ without being contained in it gives rise to a contradiction.

We have thus proved that $X$ has at least one fiber type contraction, associated to a ray $R_{2}$.

Suppose by contradiction that every other ray $R_{i}$ of $\mathrm{NE}(X)$ is birational. By lemma 4.2 (b), for the divisor $D_{1}^{2}:=\operatorname{Locus}\left(R^{2}\right)_{F_{1}}$ we have $D_{1}^{2} \cdot R^{i}=0$; moreover lemma 4.2 (a) implies that $D_{1}^{2} \cdot R^{2}=0$, so $\mathrm{NE}(X)=\left\langle R_{1}, R_{2}, R_{3}\right\rangle$.

The ray $R_{3}$ cannot be divisorial, otherwise we would have by lemma 4.2 (c) that $E_{3} \cdot R^{1}=$ $E_{3} \cdot R^{2}=0$ while $E_{3} \cdot R^{3}<0$, against the effectiveness of $E_{3}$, so $R_{3}$ must be small.

Let $F_{3}$ be an irreducible component of a fiber of $\varphi_{R_{3}}$ and let $D_{3}^{2}:=\operatorname{Locus}\left(R^{2}\right)_{F_{3}}$; by lemma 4.2 we have $D_{3}^{2} \cdot R^{1}=D_{3}^{2} \cdot R^{2}=0$.

Consider a minimal horizontal dominating family $V^{\prime}$ for the fiber type contraction $\varphi_{R_{2}}$; from the results in Section 8, Case 1, [1 we know that $V^{\prime}$ is unsplit.

The family is independent either from $R_{1}$ and $R_{2}$ or from $R_{2}$ and $R_{3}$; assume without loss of generality that we are in the first case.

If $V^{\prime}$ is covering we have $X=\operatorname{Locus}\left(V^{\prime}, R^{2}\right)_{F_{1}}=\operatorname{Locus}\left(R^{2}, V^{\prime}\right)_{F_{1}}$, so $R^{3}=V^{\prime}$ and $R_{3}$ is of fiber type, a contradiction.

If else $V^{\prime}$ is noncovering, then by remark 4.1 we have $D_{1}^{2} \cdot V^{\prime}=D_{3}^{2} \cdot V^{\prime}=0$, so that $\left[R^{2}\right]=\left[\lambda V^{\prime}\right]$, again a contradiction.

We have thus proved that $X$ admits a small ray $R_{1}$ and at least two fiber type rays $R_{2}$, $R_{3}$; by lemma 4.1 we know that the families $R^{2}, R^{3}$ are unsplit, so by lemma 2.1 we have that $X=\operatorname{Locus}\left(R^{3}, R^{2}\right)_{F_{1}}=\operatorname{Locus}\left(R^{2}, R^{3}\right)_{F_{1}}$ and lemma 2.2 implies that $\operatorname{NE}(X)=\left\langle R_{1}, R_{2}, R_{3}\right\rangle$.

Case 3. In $\operatorname{NE}(X)$ there is at least a birational ray, but no small rays.

In this case we prove that $\mathrm{NE}(X)=\left\langle R_{1}, R_{2}, R_{3}\right\rangle$, where at least one $R_{i}$ is of fiber type, and that the possible cases are the ones listed in theorem 1.1

Since $X$ has no small contractions we know by lemma 2.4 that $[V]$ lies on an extremal face of $\mathrm{NE}(X)$.

Suppose that there exists a ray $R_{1}$ which does not lie in a face with $[V]$, and denote by $E_{1}$ its exceptional locus.

If either $R_{1}$ is divisorial and $E_{1} \cdot V>0$ or $R_{1}$ is of fiber type then the associated family $R^{1}$ is 
horizontal and dominating with respect to the $\mathrm{rc} V$-fibration. Hence we can apply lemma 2.4 to $V$ and $R^{1}$ and conclude that $[V]$ and $\left[R^{1}\right]$ are on the same extremal face, a contradiction. So we can assume that $R_{1}$ is divisorial and $E_{1} \cdot V=0$. Then $E_{1}$ must be negative on another ray $R_{2}$ which lies in a face with $[V]$ : in fact, $E_{1}$ cannot vanish on a face containing $[V]$, otherwise it would be $\leq 0$ on the entire cone; clearly $R_{2}$ has to be divisorial. Then we can conclude from lemma 4.3 that in $\mathrm{NE}(X)_{E_{1}<0}$ there are two divisorial rays, in $\mathrm{NE}(X)_{E_{1}>0}$ there are only fiber type rays and $\mathrm{NE}\left(E_{1}\right)=\left\langle R_{1}, R_{2}\right\rangle$.

Let $R_{3}$ be one of the fiber type rays; we can write $X=\operatorname{Locus}\left(R^{3}\right)_{E_{1}}$, and we have by remark 2.4 that $\mathrm{NE}(X)=\left\langle R_{1}, R_{2}, R_{3}\right\rangle$.

In the case when every extremal ray lies on a face with $V$ we have trivially that $\operatorname{NE}(X)=$ $\left\langle[V], R_{1}, R_{2}\right\rangle$.

If $X$ has two fiber type rays $R_{1}, R_{2}$ and one divisorial ray $R_{3}$, then $\varphi_{R_{3}}$ cannot have a four-dimensional fiber $F_{3}$, otherwise we would have $X=\operatorname{Locus}\left(R^{1}\right)_{F_{3}}$ and $\rho_{X}=2$, by remark 2.4 .

Finally, in the case when $X$ has one fiber type ray $R_{1}$ and two divisorial rays $R_{2}$ and $R_{3}$, we claim that both $R_{2}$ and $R_{3}$ have two-dimensional fibers: in fact, if $R_{2}$ has a fiber $F_{2}$ of dimension three, by lemma 4.1 and lemma 4.2 (c) we have that $E_{3} \cdot V=E_{3} \cdot R^{2}=0$, a contradiction.

$\rho_{\boldsymbol{X}}=4$. In this case (see Section 8 , Case 2 in [1]) $X$ is rationally connected with respect to four independent unsplit families $V, V^{\prime}, V^{\prime \prime}$ and $V^{\prime \prime \prime}$, such that each one is horizontal with respect to the fibration associated to the previous ones.

By remark 2.5. for three among these families, say $V, V^{\prime}$ and $V^{\prime \prime}$, the pointed locus has dimension 1 , so these families are covering.

Moreover, if there exists a small ray $R$ we can choose two covering families, say $V$ and $V^{\prime}$, such that $[V],\left[V^{\prime}\right]$ and $[R]$ are numerically independent; then if $F$ is a fiber of $\varphi_{R}$ we can write $X=\operatorname{Locus}\left(V, V^{\prime}\right)_{F}$, implying that $\rho_{X}=3$, a contradiction. So two cases are possible: either all rays are of fiber type or there exists a divisorial ray.

Suppose that all the rays of $\mathrm{NE}(X)$ are of fiber type. If there exist two rays $R_{1}, R_{2}$ which do not lie on the same extremal face of $\mathrm{NE}(X)$, we can consider the rationally connected fibration $\pi: X-->Z$ associated to $R^{1}$ and $R^{2}$. Since $\rho_{X}=4$ we have $\operatorname{dim} Z>0$, so by lemma $2.4 X$ must have a small elementary contraction, a contradiction.

So every pair of extremal rays lies on an extremal two-dimensional face of $\mathrm{NE}(X)$; it is easy to verify that in this case $\mathrm{NE}(X)$ has exactly four rays.

Suppose now that there exists a divisorial ray $R$.

Since $X$ has no small contractions and $\rho_{X}=4, V, V^{\prime}$ and $V^{\prime \prime}$ lie on the same extremal face $\sigma$ of $\mathrm{NE}(X)$ by lemma 2.4, and, applying again lemma 2.4 to every pair of families chosen among $V, V^{\prime}$ and $V^{\prime \prime}$, we get that $\sigma=\left\langle[V],\left[V^{\prime}\right],\left[V^{\prime \prime}\right]\right\rangle$.

Let $F$ be a fiber of $\varphi_{R}$, which has dimension greater than two by proposition 2.2. Since $R$ is not in $\sigma$ we have $\operatorname{dim} \operatorname{Locus}\left(V, V^{\prime}, V^{\prime \prime}\right)_{F} \geq \operatorname{dim} F+3$ by lemma 2.1, so $\operatorname{dim} F=2$, $X=\operatorname{Locus}\left(V, V^{\prime}, V^{\prime \prime}\right)_{F}$ and every curve in $X$ can be written with positive coefficients with 
respect to $R$ and $V$; but $V, V^{\prime}$ and $V^{\prime \prime}$ play a symmetric role, so we can conclude that $\mathrm{NE}(X)=$ $\left\langle[V],\left[V^{\prime}\right],\left[V^{\prime \prime}\right],[R]\right\rangle$.

\section{FANO FIVEFOLDS WITHOUT A COVERING QUASI-UNSPLIT FAMILY}

In this section we conclude the proof of theorem 1.1 considering Fano fivefolds $X$ which do not have any covering quasi-unsplit locally unsplit family; more precisely we prove the following Theorem 1.2, Let $X$ be a Fano fivefold of pseudoindex two which does not have a covering quasi-unsplit locally unsplit family of rational curves; then $\rho_{X}=2$ and $X$ is the blow-up of $\mathbb{P}^{5}$ along a two-dimensional smooth quadric (a section of $\mathcal{O}(2)$ in a linear $\mathbb{P}^{3} \subset \mathbb{P}^{5}$ ), or along a cubic scroll $\left(\mathbb{P}_{\mathbb{P}^{1}}(\mathcal{O}(1) \oplus \mathcal{O}(2))\right.$ embedded in an hyperplane of $\mathbb{P}^{5}$ by the tautological bundle), or along a Veronese surface.

Proof. Let $V$ be a locally unsplit dominating family on $X$ and let $\mathcal{V}$ be the associated Chow family. Since $V$ is not quasi-unsplit then $[V]$ cannot be extremal; in particular it follows that $\rho_{X} \geq 2$. Moreover, since $V$ is locally unsplit but not unsplit we have

$$
4=2 i_{X} \leq \operatorname{deg} V \leq \operatorname{dim} X+1=6 ;
$$

moreover, if $\operatorname{deg} V=6$ then we would have $X=\operatorname{Locus}\left(V_{x}\right)$ for a general $x \in X$ and $\rho_{X}=1$ by corollary 2.1, a contradiction, therefore we can assume that $4 \leq \operatorname{deg} V \leq 5$. As a consequence we have that every reducible cycle in $\mathcal{V}$ splits into exactly two irreducible components.

Consider the pairs $\left(W^{i}, \bar{W}^{i}\right)$ of families such that there is a cycle in $\mathcal{V}$ whose irreducible components belong respectively to $W^{i}$ and $\bar{W}^{i}$, and let $\mathcal{B}$ be the set of these pairs. By this definition we clearly have $\left[W^{i}\right]+\left[\bar{W}^{i}\right]=[V]$, and since the anticanonical degree of these families is bounded they are only a finite number.

We begin establishing some properties of these pairs.

Lemma 5.1. If $\left(W^{i}, \bar{W}^{i}\right) \in \mathcal{B}$ then the families $W^{i}, \bar{W}^{i}$ are unsplit, and moreover $\left(\operatorname{dim} \operatorname{Locus}\left(W^{i}\right), \operatorname{dim} \operatorname{Locus}\left(W_{x}^{i}\right)\right)$ is either $(4,2),(4,3),(4,4)$ or $(3,3)$.

Proof. The families are unsplit since

$$
4=2 i_{X} \leq \operatorname{deg} W^{i}+\operatorname{deg} \bar{W}^{i}=\operatorname{deg} V \leq 5,
$$

and therefore they are noncovering, so the second assertion follows from proposition 2.1 .

Lemma 5.2. Let $\left(W^{i}, \bar{W}^{i}\right) \in \mathcal{B}$ such that $\left[W^{i}\right] \neq[\alpha V]$, and let $D_{i}$ and $\bar{D}_{i}$ be meeting components of $\operatorname{Locus}\left(W^{i}\right)$ and $\operatorname{Locus}\left(\bar{W}^{i}\right)$. Up to exchange $D_{i}$ and $\bar{D}_{i}$, we have that $\left(\operatorname{dim} D_{i}, \operatorname{dim} \bar{D}_{i}\right)$ is either $(3,4)$ or $(4,4)$.

Proof. By lemma 5.1 we have $\operatorname{dim} D_{i}, \operatorname{dim} \bar{D}_{i} \geq 3$, and equality holds if and only if $D_{i}=$ $\operatorname{Locus}\left(W_{x}^{i}\right)$ for some $x$; in this case $N_{1}\left(D_{i}\right)=\left\langle\left[W^{i}\right]\right\rangle$ by corollary 2.1]

So if $\operatorname{dim} D_{i}=\operatorname{dim} \bar{D}_{i}=3$ we have $\operatorname{dim}\left(D_{i} \cap \bar{D}_{i}\right) \geq 1$, contradicting the fact that $N_{1}\left(D_{i}\right)=$ $\left\langle\left[W^{i}\right]\right\rangle$ and $N_{1}\left(\bar{D}_{i}\right)=\left\langle\left[\bar{W}^{i}\right]\right\rangle$. 
Lemma 5.3. Let $R_{1}$ be a divisorial ray of $X$, and $E_{1}$ its exceptional locus. If there exists a pair $\left(W^{i}, \bar{W}^{i}\right) \in \mathcal{B}$ such that $E_{1} \cdot W^{i}<0$ and $E_{1} \cdot \bar{W}^{i}>0$, then $\left[W^{i}\right] \in R_{1}$.

Proof. Since $E_{1} \cdot W^{i}<0$ we have $\operatorname{Locus}\left(W^{i}\right) \subset E_{1}$; suppose by contradiction that $\left[W^{i}\right] \notin R_{1}$.

If $\operatorname{dim} \operatorname{Locus}\left(W_{x}^{i}\right) \quad \geq 3$ for some $x$, then by lemma 2.1 (a) we have $\operatorname{dim} \operatorname{Locus}\left(W^{i}, R^{1}\right)_{x} \geq 5$, a contradiction since $W^{i}$ is noncovering.

It follows that $\operatorname{dim} \operatorname{Locus}\left(W_{x}^{i}\right)=2$ and so, by lemma 5.1 $\operatorname{dim} \operatorname{Locus}\left(W^{i}\right)=4$, hence $E_{1}=$ $\operatorname{Locus}\left(W^{i}\right)=\operatorname{Locus}\left(R^{1}, W^{i}\right)_{x}$ for some $x$; in particular by corollary 2.2

$$
\mathrm{NE}\left(E_{1}\right)=\left\langle R_{1},\left[W^{i}\right]\right\rangle \subset \mathrm{NE}(X)_{E_{1}<0} .
$$

On the other hand, since $\operatorname{dim} \operatorname{Locus}\left(\bar{W}_{x}^{i}\right) \geq 2$ and $E_{1} \cdot \bar{W}^{i}>0$, we have that $E_{1}$ contains curves proportional to $\bar{W}^{i}$, a contradiction.

We can now resume the proof of theorem 1.2

Step 1. $\operatorname{deg} V=4$.

Suppose by contradiction that $\operatorname{deg} V=5$, let $x \in X$ be a general point and let $D$ be an irreducible component of $\operatorname{Locus}\left(V_{x}\right)$; since $V$ is locally unsplit, by corollary 2.1 we have $N_{1}(D)=\langle[V]\rangle$, and by proposition 2.1 we have $\operatorname{dim} D \geq \operatorname{deg} V-1 \geq 4$.

We are assuming $\rho_{X} \geq 2$, so it cannot be $D=X$, therefore $D$ is an effective divisor.

Thus the rc $\mathcal{V}$-fibration $\pi: X-->Z$ has fibers of dimension $\geq 4$; if $Z$ has positive dimension, take $V^{\prime}$ to be a horizontal dominating family for $\pi$. By remark 2.5 we know that $\operatorname{dim} \operatorname{Locus}\left(V_{x}^{\prime}\right)=1$, so $V^{\prime}$ is covering and of degree 2, hence it is unsplit, a contradiction.

So $X$ is rc $\mathcal{V}$-connected; in particular $N_{1}(X)$ is generated as a vector space by the numerical class of $V$ and the numerical classes of the families $W^{i}$ such that $\left(W^{i}, \bar{W}^{i}\right) \in \mathcal{B}$ for some $\bar{W}^{i}$. Consider the nonempty set of pairs $\left(W^{i}, \bar{W}^{i}\right) \in \mathcal{B}$ such that $\left[W^{i}\right] \neq[\alpha V]$, and the (non negative) intersection number $D \cdot V$.

If $D \cdot V=0$ then $D$ would be negative on at least one of these $W^{i}$ and so it would contain curves in $W^{i}$, against the fact that $N_{1}(D)=\langle[V]\rangle$.

If else $D \cdot V>0$ then for every $i$ either $D \cdot W^{i}>0$ or $D \cdot \bar{W}^{i}>0$; but in this case either $\operatorname{Locus}\left(W_{x}^{i}\right) \cap D$ or $\operatorname{Locus}\left(\bar{W}_{x}^{i}\right) \cap D$ would be nonempty.

Suppose without loss of generality that we are in the first case; since, by lemma 5.1, $\operatorname{dim} \operatorname{Locus}\left(W_{x}^{i}\right) \geq$ 2 , then $\operatorname{dim}\left(\operatorname{Locus}\left(W_{x}^{i}\right) \cap D\right) \geq 1$, against the fact that $N_{1}\left(\operatorname{Locus}\left(W_{x}^{i}\right)\right)=\left\langle\left[W^{i}\right]\right\rangle$ and $N_{1}(D)=$ $\langle[V]\rangle$.

As a corollary of step 1 we get that $V$ is the unique locally unsplit dominating family for $X$ up to numerical equivalence: in fact, if $V^{\prime}$ were another locally unsplit dominating family, for the general point $x \in X$ we would have $\operatorname{dim}\left(\operatorname{Locus}\left(V_{x}\right) \cap \operatorname{Locus}\left(V_{x}^{\prime}\right)\right) \geq 1$ and so, since $N_{1}\left(\operatorname{Locus}\left(V_{x}\right)\right)=\langle[V]\rangle$ and $N_{1}\left(\operatorname{Locus}\left(V_{x}^{\prime}\right)\right)=\left\langle\left[V^{\prime}\right]\right\rangle$, the families would be proportional. But we have proved that $\operatorname{deg} V=\operatorname{deg} V^{\prime}=4$, so $[V]=\left[V^{\prime}\right]$.

Step 2 If $R_{1}$ is a small ray, then $\left[R^{1}\right]=\left[W^{i}\right]$ for some $i$. 
By the results in Section 9, 1, we know that either $X$ is $\mathrm{rc} \mathcal{V}$-connected or there exists an unsplit noncovering family $V^{\prime} \operatorname{such}$ that $\operatorname{deg} V^{\prime}=2, \operatorname{dim} \operatorname{Locus}\left(V_{x}^{\prime}\right)=2$ (and so, by proposition $\left.2.1 \operatorname{dim} \operatorname{Locus}\left(V^{\prime}\right)=4\right)$ and $X$ is $\operatorname{rc}\left(\mathcal{V}, V^{\prime}\right)$-connected.

In particular, through every point of a fiber $F_{1}$ of $\varphi_{R_{1}}$ there passes a curve in $V$, in $W^{i}$ or in $V^{\prime}$; if $V_{y}$ is unsplit for some $y \in F_{1}$ then $\operatorname{dim}\left(\operatorname{Locus}\left(V_{y}\right) \cap F_{1}\right) \geq 1$, against the fact that $N_{1}\left(V_{y}\right)=\langle[V]\rangle$ and $N_{1}\left(F_{1}\right)=\left\langle R_{1}\right\rangle$.

Therefore through every $y \in F_{1}$ there passes either a curve in $V^{\prime}$ or a reducible cycle of $\mathcal{V}$; recalling that in $\mathcal{B}$ there is only a finite number of families we have that either $F_{1} \subset \operatorname{Locus}\left(V^{\prime}\right)$ or $F_{1} \subset \operatorname{Locus}\left(W^{i}\right)$ for some $i$.

Suppose first that $F_{1} \subset \operatorname{Locus}\left(V^{\prime}\right)$; note that, since $\operatorname{Locus}\left(V^{\prime}\right)$ has dimension four and $R_{1}$ is a small ray, the numerical class $\left[V^{\prime}\right]$ cannot belong to $R_{1}$, hence $R^{1}$ is independent from $V^{\prime}$ and we can apply lemma 2.1 (a) and obtain that $\operatorname{dim} \operatorname{Locus}\left(V^{\prime}\right)_{F_{1}} \geq 5$, a contradiction.

If else $F_{1} \subset \operatorname{Locus}\left(W^{i}\right)$ and $R^{1}$ is independent from $W^{i}$ we reach a contradiction again by lemma 2.1 (a), so $\left[W^{i}\right]=\left[\alpha R^{1}\right]$.

By step 1 we know that $\operatorname{deg} W^{i}=2$, and since $R^{1}$ has minimal degree in the ray we have also $\operatorname{deg} R^{1}=2$, i.e. $\left[R^{1}\right]=\left[W^{i}\right]$.

Step $3 \quad \rho_{X}=2$.

If $X$ is not $\mathrm{rc} \mathcal{V}$-connected then the result follows from Section 9, Case 1 in [1].

Assume now that $X$ is rc $\mathcal{V}$-connected.

By the results in Section 9 of [1], if $\rho_{X} \geq 3$ then for every pair $\left(W^{i}, \bar{W}^{i}\right) \in \mathcal{B}$ and meeting components $D_{i}$ and $\bar{D}_{i}$ of $\operatorname{Locus}\left(W^{i}\right)$ and $\operatorname{Locus}\left(\bar{W}^{i}\right)$ we have $\operatorname{dim} D_{i}=\operatorname{dim} \bar{D}_{i}=4$; moreover for at least one pair, say $\left(W^{1}, \bar{W}^{1}\right)$, we know that $D_{1} \neq \bar{D}_{1}$. In particular $X$ has no small contractions by step 2 .

Let $E_{1}$ be the exceptional locus of a (divisorial) ray $R_{1}$ of $X$ and consider the intersection number $E_{1} \cdot V$.

If $E_{1} \cdot V>0$, for a general point $x \in X$ we have $\operatorname{dim} \operatorname{Locus}\left(R^{1}\right)_{\operatorname{Locus}\left(V_{x}\right)}=4$, so that $E_{1}=\operatorname{Locus}\left(R^{1}\right)_{\operatorname{Locus}\left(V_{x}\right)}$ and $N_{1}\left(E_{1}\right)=\left\langle R_{1},[V]\right\rangle$ by lemma 2.2 .

If every pair $\left(W^{i}, \bar{W}^{i}\right)$ lies in the plane spanned by $[V]$ and $\left[R^{1}\right]$ then $\rho_{X}=2$ and we conclude, otherwise let $\left(W^{i}, \bar{W}^{i}\right)$ be a pair not lying in that plane: then either $E_{1} \cdot W^{i}>0$ or $E_{1} \cdot \bar{W}^{i}>0$, implying that either $E_{1} \cap \operatorname{Locus}\left(W_{x}^{i}\right)$ or $E_{1} \cap \operatorname{Locus}\left(\bar{W}_{x}^{i}\right)$ is nonempty and so has dimension $\geq 1$, a contradiction.

If else $E_{j} \cdot V=0$ for every $j$, then for every $j$ there exists $i$ such that $E_{j} \cdot W^{i}<0$ and $E_{j} \cdot \bar{W}^{i}>0$, so by lemma $5.3\left[W^{i}\right] \in R_{j}$ for some $i$ and $E_{j}=D_{i}$.

Let $R_{k}$ be an extremal ray such that $D_{i} \cdot R^{k}>0$; by the argument above we know that $E_{k}=\bar{D}_{l}$ for some $l$, so it must be $D_{i} \cdot W^{l}<0$ and $i=l$, i.e. $E_{k}=\bar{D}_{i}$.

It follows that $D_{i} \cdot V=\bar{D}_{i} \cdot V=0$, but this is excluded in Section 9, Case 2b of 1] (note that since $D_{i} \cdot R^{k}>0$ we have $D_{i} \neq E_{k}=\bar{D}_{i}$ ).

Step $4 \quad X$ has a divisorial contraction. 
Suppose that both the rays $R_{1}$ and $R_{2}$ correspond to small contractions; by step 2 we know that there exist unsplit families $W^{1}$ and $W^{2}$ such that $\left(R^{1}, W^{1}\right),\left(R^{2}, W^{2}\right) \in \mathcal{B}$, so in particular $[V]=\left[R^{1}\right]+\left[W^{1}\right]=\left[R^{2}\right]+\left[W^{2}\right]$.

Let $x_{1} \in \operatorname{Locus}\left(R^{1}\right)$ and $x_{2} \in \operatorname{Locus}\left(R^{2}\right)$; since the contractions associated to $R^{1}$ and $R^{2}$ are small, by inequality 2.2 we have $\operatorname{dim} \operatorname{Locus}\left(R^{i}\right)_{x_{i}} \geq 3$.

Denote by $D_{i}$ an irreducible component of $\operatorname{Locus}\left(R^{i}, W^{i}\right)_{x_{i}}$; since

$\operatorname{Locus}\left(R^{i}, W^{i}\right)_{x_{i}}=\operatorname{Locus}\left(W^{i}\right)_{\operatorname{Locus}\left(R^{i}\right)_{x_{i}}}$, by lemma 2.1 (b) we have $\operatorname{dim} D_{i}=4$, and by corollary 2.2 we have $\operatorname{NE}\left(D_{1}\right)=\left\langle R_{1},\left[W^{1}\right]\right\rangle, \operatorname{NE}\left(D_{2}\right)=\left\langle R_{2},\left[W^{2}\right]\right\rangle$.

It follows that $D_{1} \cdot R^{2}=D_{2} \cdot R^{1}=0$; moreover, since $D_{i}$ is an effective divisor for every $i$, we have $D_{i} \cdot R^{i}>0$, so $D_{i}$ is nef.

Write $-K_{X}=a D_{1}+b D_{2}$; we have

$$
\begin{aligned}
4 & =-K_{X} \cdot\left(W^{1}+W^{2}\right)= \\
& =a D_{1} \cdot\left(W^{1}+W^{2}+R^{2}\right)+b D_{2} \cdot\left(W^{1}+W^{2}+R^{1}\right)= \\
& =a D_{1} \cdot W^{1}+a D_{1} \cdot V+b D_{2} \cdot W^{2}+b D_{2} \cdot V= \\
& =a D_{1} \cdot W^{1}+b D_{2} \cdot W^{2}-K_{X} \cdot V .
\end{aligned}
$$

Hence $a D_{1} \cdot W^{1}=b D_{2} \cdot W^{2}=0$, a contradiction.

Step 5 There exists a ray $R_{1}$ such that its associated contraction $\varphi_{1}: X \rightarrow Y$ is a smooth blow-up of $Y$ along a smooth surface; moreover, if $E_{1}$ is the exceptional divisor, $E_{1} \cdot V>0$.

We know by step 4 that $X$ has a divisorial ray $R_{1}$; the other ray $R_{2}$ can be either small or divisorial.

Let us start assuming that $R_{2}$ is small; denote by $E_{1}$ the exceptional locus of $R_{1}$ and by $G_{2}$ a component of the exceptional locus of $R_{2}$.

The divisor $E_{1}$ is positive on $R^{2}$; it follows that $\operatorname{Locus}\left(R^{1}\right)_{G_{2}}$ is nonempty and has dimension four, so that $E_{1}=\operatorname{Locus}\left(R^{1}\right)_{G_{2}}$; in particular every fiber of $R_{1}$ meets $G_{2}$ and so it is twodimensional.

We can thus apply Theorem 5.1 of [2] and we get that $\varphi_{1}: X \rightarrow Y$ is a blow-down with center a smooth surface $S$.

By step 2 there exists a pair $\left(W^{1}, \bar{W}^{1}\right) \in \mathcal{B}$ such that $\left[\bar{W}^{1}\right]=\left[R^{2}\right]$. Take $D_{1}$ to be an irreducible component of $\operatorname{Locus}\left(W^{1}\right)$ which intersects $\operatorname{Locus}\left(\bar{W}^{1}\right)$; since $D_{1} \cdot \bar{W}^{1}>0$ we have that $D_{1}=\operatorname{Locus}\left(\bar{W}^{1}, W^{1}\right)_{x}$ and so $\operatorname{NE}\left(D_{1}\right)=\left\langle\left[W^{1}\right],\left[\bar{W}^{1}\right]\right\rangle$.

We claim that $\left[W^{1}\right]=\left[R^{1}\right]$ : if this is not the case then $D_{1} \neq E_{1}$, so $\varphi_{1}\left(D_{1}\right)$ is an effective divisor on $Y$. Moreover $\varphi_{1}\left(D_{1}\right)$ is ample since $\rho_{Y}=1$, hence it meets $S$ and $D_{1} \cap E_{1} \neq \emptyset$.

It follows that $\operatorname{dim}\left(D_{1} \cap \operatorname{Locus}\left(R_{x}^{1}\right)\right) \geq 1$ and $D_{1}$ contains curves numerically proportional to $R^{1}$, a contradiction.

So we have proved that $\mathrm{NE}(X)=\left\langle R_{1}, R_{2}\right\rangle=\left\langle\left[W^{1}\right],\left[\bar{W}^{1}\right]\right\rangle$; moreover we have that $\operatorname{Locus}\left(W^{1}\right)=$ $E_{1}$. 
Now we show that $E_{1} \cdot V>0$.

Suppose first that $X$ is not $\mathrm{rc} \mathcal{V}$-connected and denote by $\pi$ the $\operatorname{rc} \mathcal{V}$-fibration. Let $X^{0}$ be the open subset on which $\pi$ is defined, take $x \in E_{1} \cap X^{0}$ and consider $\operatorname{Locus}\left(R_{x}^{1}\right) ; \operatorname{since} \operatorname{dim} Z \leq 2$ either $\operatorname{Locus}\left(R_{x}^{1}\right)$ is contained in a fiber of $\pi$ or $\operatorname{Locus}\left(R_{x}^{1}\right)$ dominates $Z$.

In the first case, if $H$ is an ample divisor on $Z$ we have that $\left(\pi^{*} H\right) \cdot V=\left(\pi^{*} H\right) \cdot R^{1}=0$, so $\pi^{*} H$ is numerically trivial on $X$, a contradiction, hence $\operatorname{Locus}\left(R_{x}^{1}\right)$ dominates $Z$ and $\operatorname{dim} Z=2$.

For a general $x$ in $X$ the fiber of $\pi$ through $x$ has dimension three and contains $\operatorname{Locus}\left(V_{x}\right)$, hence $F_{x}=\operatorname{Locus}\left(V_{x}\right) ; E_{1}$ meets this fiber and cannot contain it, so $E_{1} \cdot V>0$.

Assume now that $X$ is $\mathrm{rc} \mathcal{V}$-connected and suppose by contradiction that $E_{1} \cdot V=0$; in this case, by lemma $5.3 \mathcal{B}$ contains only the pair $\left(W^{1}, \bar{W}^{1}\right)$ and possibly pairs $\left(W^{j}, \bar{W}^{j}\right)$ with $\left[W^{j}\right]=\left[\bar{W}^{j}\right]=\frac{1}{2}[V]$.

Let $T=\operatorname{Locus}\left(W^{1}\right) \cup \operatorname{Locus}\left(\bar{W}^{1}\right)$ and take a point $x$ outside $T$; since $X$ is rc $\mathcal{V}$-connected we can join $x$ and $T$ with a chain of cycles in $\mathcal{V}$. Let $\Gamma$ be the first irreducible component which meets $T$.

Clearly $\Gamma$ cannot belong to $\left[W^{1}\right]$ and $\left[\bar{W}^{1}\right]$ because it is not contained in $T$, so it belongs either to $V$ or to $W^{j}$ for some $j$, say $j=2$. Since $E_{1} \cdot V=E_{1} \cdot W^{2}=0, \Gamma$ must intersect $T$ in points of $T \backslash \operatorname{Locus}\left(W^{1}\right)$.

Let $y$ be a point in $\Gamma \cap T$ and let $G_{y}$ be the irreducible component of $T$ which contains $y$; by Lemma 9.1 in [1] we have that either $\Gamma \subset \operatorname{Locus}\left(V_{z}\right)$ for some $z$ such that $V_{z}$ is unsplit or $\Gamma \subset \operatorname{Locus}\left(W^{2}\right)$.

In the first case we have $\operatorname{dim}\left(\operatorname{Locus}\left(V_{z}\right) \cap G_{y}\right) \geq 1$, against the fact that $N_{1}\left(V_{z}\right)=\langle[V]\rangle$ and $N_{1}\left(G_{y}\right)=\left\langle\left[\bar{W}^{1}\right]\right\rangle$.

In the second case we consider $H=\operatorname{Locus}\left(W^{2}\right)_{G_{y}}$ : by lemma 2.1 we have $\operatorname{dim} H=4$, and by lemma 2.2 we have $\mathrm{NE}(H)=\left\langle\left[\bar{W}^{1}\right],\left[W^{2}\right]\right\rangle$; this implies that $H \cdot R^{1}=0$.

The image $\varphi_{1}(H)$ of $H$ in $Y$ is an effective, hence ample, divisor; therefore $\varphi_{1}(H) \cap S \neq \emptyset$ and $H \cap E_{1} \neq \emptyset$.

For every point $t \in H \cap E_{1}$ we have that both $\operatorname{Locus}\left(W_{t}^{2}\right)$ and $\operatorname{Locus}\left(W_{t}^{1}\right)$ are contained in $H \cap E_{1}$, since $H \cdot W^{1}=E_{1} \cdot W^{2}=0$.

Therefore $\operatorname{Locus}\left(W^{2}, W^{1}\right)_{t} \subseteq H \cap E_{1}$, and we reach a contradiction since $\operatorname{dim} \operatorname{Locus}\left(W^{2}, W^{1}\right)_{t}=$ 4 by lemma 2.1 (a).

Assume now that both $R_{1}$ and $R_{2}$ are divisorial and let $E_{1}, E_{2}$ be the respective exceptional loci.

We cannot have $E_{1} \cdot V=E_{2} \cdot V=0$ (see the end of proof of Theorem 7.1 in [1]), so we can suppose that $E_{1} \cdot V>0$.

If $x \in X$ is a general point then $\operatorname{Locus}\left(R^{1}\right)_{\operatorname{Locus}\left(V_{x}\right)}$ is nonempty and has dimension four, so $E_{1}=\operatorname{Locus}\left(R^{1}\right)_{\operatorname{Locus}\left(V_{x}\right)}$; in particular every fiber of $R_{1} \operatorname{meets} \operatorname{Locus}\left(V_{x}\right)$ and so it is twodimensional.

Now we apply Theorem 5.1 of [2] and we get that $\varphi_{1}: X \rightarrow Y$ is a blow-down with center a smooth surface $S$. 
Step $6 \quad Y \simeq \mathbb{P}^{5}$.

Let $V_{Y}$ be a minimal covering family for $Y$ and let $V_{Y}^{*}$ be the family of deformations of the strict transform of a general curve in $V_{Y}$. We know that $V_{Y}^{*}$ is covering and that

$$
6=\operatorname{dim} Y+1 \geq-\varphi_{1}^{*} K_{Y} \cdot V_{Y}^{*}=-K_{X} \cdot V_{Y}^{*}+2 E_{1} \cdot V_{Y}^{*} .
$$

By Proposition 3.7 in [12] a general member of $V_{Y}$ does not meet $S$, which has codimension three in $Y$, hence $E_{1} \cdot V_{Y}^{*}=0$.

The family $V_{Y}^{*}$ cannot be locally unsplit: otherwise, by the final part of step one, we would have $\left[V_{Y}^{*}\right]=[V]$, but we know by step 5 that $E_{1} \cdot V>0$.

It follows that one among the families of irreducible components of cycles in $\mathcal{V}_{Y}^{*}$ is covering, and so, by our assumptions, the degree of this family is at least four; in particular $-K_{X} \cdot V_{Y}^{*} \geq 6$. Equation 5.1 yields

$$
6 \geq-K_{Y} \cdot V_{Y}-\varphi_{1}^{*} K_{Y} \cdot V_{Y}^{*}=-K_{X} \cdot V_{Y}^{*} \geq 6,
$$

so we can conclude that $Y$ has a minimal (hence locally unsplit by lemma 2.3) covering family of degree $6=\operatorname{dim} Y+1$; by the proof of Theorem 1.1 in [10] we have $Y \simeq \mathbb{P}^{5}$. (Note that the assumptions of the quoted result are different, but the proof actually works in our case since for a very general $y$ the pointed family $\left(V_{Y}\right)_{y}$ has the properties 1-3 in Theorem 2.1 of [10]).

\section{Final step}

Let $S \subset \mathbb{P}^{5}$ be the center of the blow-up, let $l$ be a (bi)secant line of $S$ and let $\tilde{l}$ be the proper transform of $l$; then

$$
-K_{X} \cdot \tilde{l}=\varphi_{1}^{*} \mathcal{O}(6) \cdot \tilde{l}-2 E_{1} \cdot \tilde{l}=2,
$$

so the corresponding family on $X$ is unsplit. Since $X$ does not admit unsplit covering families, through the general point of $\mathbb{P}^{5}$ there is no secant line of $S$.

It follows that either $S$ is a Veronese surface or $S$ is degenerate.

If $S$ is contained in an hyperplane $H$ and in no three-dimensional linear subspace of $\mathbb{P}^{5}$, through every point of $H$ there is a secant line, so the strict transform $\widetilde{H}$ of $H$ is the locus of an unsplit family $W$ on $X$.

Since $\varphi_{1}^{*} \mathcal{O}(1)=\varphi_{1}^{*} H=\widetilde{H}+k E_{1}$ with $k>0$ we have $\widetilde{H}=\varphi_{1}^{*} \mathcal{O}(1)-k E_{1}$, so $\widetilde{H} \cdot W<0$. It follows that $\widetilde{H}$ is negative on $R_{2}$ and it follows that $\widetilde{H}=E_{2}, R_{2}$ is divisorial and $W=R^{2}$ by lemma 5.3 .

Again from the canonical bundle formula we have $E_{1} \cdot R^{2}=2$, so $k=1$ and $E_{2}=\widetilde{H}=$ $\varphi_{1}^{*} \mathcal{O}(1)-E_{1}$; in particular $\varphi_{1}^{*} \mathcal{O}(1) \cdot R^{2}=1$ and the contraction $\varphi_{2}: X \rightarrow Z$ is supported by $K_{X}+2 \varphi_{1}^{*} \mathcal{O}(1)+\varphi_{2}^{*} A$ for some very ample divisor $A$ on $Z$. By Corollary 5.8.1 of [4] $\varphi_{2}$ is equidimensional, so it is a smooth blow-down.

Computing the canonical bundle of $E_{2}$

$$
K_{E_{2}}=-5 \varphi_{1}^{*} \mathcal{O}(1)+E_{1},
$$

we find that $E_{2}$ is a Fano variety.

Moreover $E_{2}$ has a $\mathbb{P}^{2}$-bundle structure over a smooth surface $S^{\prime} \subset Z$, and since $\rho\left(E_{2}\right)=2$ we 
have $S^{\prime} \simeq \mathbb{P}^{2}$; by the classification in [19] we know that $S$ is a cubic scroll.

Finally, if $S$ is contained in a three-dimensional linear subspace $\Lambda \subset \mathbb{P}^{5}$ and $l$ is a line in $\Lambda$ we have

$$
-K_{X} \cdot \tilde{l}=\varphi_{1}^{*} \mathcal{O}(6) \cdot \tilde{l}-2 E_{1} \cdot \tilde{l}=6-2 \operatorname{deg} S
$$

so $S$ has degree $\leq 2$ and it cannot be a plane, since the blow-up of $\mathbb{P}^{5}$ along a two-dimensional plane has a fiber type contraction.

\section{The HigheR-Dimensional CASE}

Let $V$ be a covering locally unsplit family of rational curves on a Fano variety $X$ of dimension $n \geq 6$ and pseudoindex $i_{X}=n-3$; as shown in the case of fivefolds, if $\rho_{X} \geq 2$ then $\operatorname{deg} V \leq n$.

Lemma 6.1. Let $X$ be a Fano variety of dimension $n \geq 6$, pseudoindex $i_{X}=n-3$ and Picard number $\rho_{X} \geq 2$; let $V$ be a covering locally unsplit family of rational curves on $X$. Then $V$ is unsplit.

Proof. If $\operatorname{deg} V \leq n-1<2 i_{X}$ then $V$ is unsplit, so we can assume that $\operatorname{deg} V=n$. Let $x \in X$ be a general point and let $D$ be an irreducible component of $\operatorname{Locus}\left(V_{x}\right)$; since $V$ is locally unsplit we have $N_{1}(D)=\langle[V]\rangle$ by corollary 2.1 and, by proposition 2.1 $\operatorname{dim} D \geq \operatorname{deg} V-1=n-1$. We are assuming $\rho_{X} \geq 2$, so it cannot be $D=X$, therefore $D$ is an effective divisor.

The rc $\mathcal{V}$-fibration $\pi: X-->Z$ has fibers of dimension $\geq n-1$; if $Z$ has positive dimension, take $V^{\prime}$ to be a horizontal dominating family. Then if $F$ is a fiber of $\pi$ we have

$$
\operatorname{dim}\left(F \cap \operatorname{Locus}\left(V_{x}^{\prime}\right)\right) \geq n-1+\operatorname{deg} V^{\prime}-1-n \geq i_{X}-2 \geq 1,
$$

contradicting lemma 2.3. So $X$ is $\mathrm{rc} \mathcal{V}$-connected, and we reach a contradiction as in step 1 of section 5 ,

Corollary 6.1. In the above assumptions, Mukai conjecture (1.1) holds for X. In particular if $\operatorname{dim} X \geq 8$ then $\rho_{X}=1$ except if $X \simeq \mathbb{P}^{4} \times \mathbb{P}^{4}$.

Proof. Since $i_{X} \geq \frac{n+3}{3}$ we can apply Theorem 7.1 in [1].

To conclude the proof of theorem 1.1 we have to deal with varieties of dimension 6 and 7 . Note that Mukai conjecture implies that $\rho_{X} \leq 2$ except if $X \simeq \mathbb{P}^{2} \times \mathbb{P}^{2} \times \mathbb{P}^{2}$.

Arguing as in case $\rho_{X}=2$ of section 4 we can prove that $X$ has at least one fiber type ray $R_{1}$. Let $R_{2}$ be the other extremal ray; then if $F_{i}$ is a general fiber of the contraction $\varphi_{R_{i}}$ we know that

$$
\operatorname{dim} F_{1}+\operatorname{dim} F_{2} \leq \operatorname{dim} X,
$$

and together with the fiber locus inequality this concludes the classification. 


\section{ACKNOWLEDGEMENTS}

We thank Marco Andreatta, Edoardo Ballico, Massimiliano Mella and Roberto Pignatelli for helpful remarks and discussions. We also thank the referee for pointing out some inaccuracies in the original version.

\section{REFERENCES}

1. M. Andreatta, E. Chierici and G. Occhetta. Generalized Mukai conjecture for special Fano varieties. Central European Journal of Math. 2 (2004), 272-293.

2. M. Andreatta and G. Occhetta. Special rays in the Mori cone of a projective variety. Nagoya Math. J. 168 (2002), 127-137.

3. M. Andreatta and G. Occhetta. Fano manifolds with long extremal rays. Asian J. Math. 9 (2005), 523-544.

4. M. Andreatta and J. A. Wiśniewski. On contractions of smooth varieties. J. Algebraic Geom. 7 (1998), 253-312.

5. F. Campana. Connexité rationnelle des variétés de Fano. Ann. Sci. École Norm. Sup. 25 (1992), 539-545.

6. O. Debarre. Higher-Dimensional Algebraic Geometry, Universitext Springer, 2001.

7. L. Ein and N. Shepherd-Barron. Some special Cremona transformations. Amer. Jour. Math. 111 (1989), 783-800.

8. T. Fujita. On the structure of polarized manifolds with total deficiency one, II. J. Math. Soc. Japan 33 (1981), 415-434.

9. T. Fujita. Classification theories of polarized varieties, volume 155 of London Math. Soc. Lecture Note Series, Cambridge Univ. Press, 1990.

10. S. Kebekus. Characterizing the projective space after Cho, Miyaoka and Shepherd-Barron. In Complex geometry (Göttingen, 2000), Springer, 2002, 147-155.

11. S. Kobayashi and T. Ochiai Characterizations of complex projective spaces and hyperquadrics, J. Math. Kyoto Univ. 13 (1973), 31-47.

12. J. Kollár. Rational Curves on Algebraic Varieties, volume 32 of Ergebnisse der Math., Springer-Verlag, 1996.

13. J. Kollár, Y. Miyaoka and S. Mori. Rational connectedness and boundedness of Fano manifolds. J. Diff. Geom. 36 (1992), 765-779.

14. M. Mella. Existence of good divisors on Mukai varieties. J. Algebraic Geom. 8 (1999), 197-206.

15. S. Mukai. Open problems. In The Taniguchi foundation, editor, Birational geometry of algebraic varieties., Katata, 1988.

16. S. Mukai. Biregular classification of Fano 3-folds and Fano manifolds of coindex 3. Proc. Nat. Acad. Sci. U.S.A. 86 (1989), 3000-3002.

17. S. Nakano On the inverse of monoidal transformation. Publ. Res. Inst. Math. Sci. 6 (1970/71), 483-502.

18. G. Occhetta. A characterization of products of projective spaces. Preprint, http://www.science.unitn.it/ occhetta, to appear on Canad. Math. Bull.

19. M. Szurek and J. A. Wiśniewski. On Fano manifolds, which are $\mathbb{P}^{k}$-bundles over $\mathbb{P}^{2}$. Nagoya Math. J. 120 (1990), 89-101.

20. J. A. Wiśniewski. Fano 4-folds of index 2 with $b_{2} \geq 2$. A contribution to Mukai classification. Bull. Polish Acad. Sci. Math. 38 (1990), 173-184.

21. J. A. Wiśniewski. On a conjecture of Mukai. Manuscripta Math. 68 (1990), 135-141.

Dipartimento di Matematica, via Sommarive 14, I-38050 Povo (TN)

E-mail address: e.chierici@email.it, gianluca.occhetta@unitn.it 